\title{
$\begin{array}{ll}\text { Research Square } & \begin{array}{l}\text { Preprints are preliminary reports that have not undergone peer review. } \\ \text { They should not be considered conclusive, used to inform clinical practice, } \\ \text { or referenced by the media as validated information. }\end{array}\end{array}$
}

\section{Relationship Between Combining Ability, Genetic Components And Genetic Diversity Using Triple Test Cross}

Yasser Mohamed El-Mansy

Cotton Research Institute

Ahmed Mohamed Abdelmoghny ( $\square$ elkomy_a@yahoo.com )

Cotton Research Institute https://orcid.org/0000-0002-9915-3052

Reham. H. A. Gibely

Cotton Research Institute

Adel H. Mabrouk

Cotton Research Institute

\section{Research}

Keywords: Cotton, Triple Test Cross, Genetic diversity, Combining ability, Additive, Dominance, Epistasis, Genetic correlation

Posted Date: June 11th, 2020

DOI: https://doi.org/10.21203/rs.3.rs-33812/v1

License: (c) (i) This work is licensed under a Creative Commons Attribution 4.0 International License. Read Full License 


\section{Abstract}

Background: The target of this study is to investigate the relationship between genetic components, combining ability and genetic diversity among twenty six cotton crosses derived from a cross between thirteen contrasting inbred lines with two testers in three replications using modified triple test cross model.

Results: All the genotypes showed highly significant differences for twelve yield and fiber quality traits reflecting genetic variability between lines, testers and crosses. Giza 86 is considered as a good combiner for yield and its components traits, while Giza 45 is the best combiner for fiber fineness and fiber strength, which classified in unique cluster. Most of the combinations having significant SCA effects were belonging to genetically diverse parents. The mean squares for the deviations revealed the presence of significant epistasis for all the studied traits except, seed index and fiber reflectance. While, significant mean squares were shown for sums and differences except lint index for additive and uniformity ratio and fiber reflectance for dominance. The fixable type (i) of epistasis was larger than non-fixable $(i+I)$ type for the inheritance of the studied traits. The traits had significant mean squares for both fixable and non-fixable gene action, also showed significant GCA and SCA among 15 parents and 26 cotton crosses, respectively. Additive genetic component was larger than dominance for all the studied traits. So, most of the studied traits had significant GCA and degree of dominance was less than unity for all the studied traits, indicating partial or incomplete dominance. The correlation coefficient between the sums and difference were found to be insignificant indicating the genes with positive and negative effects were equally distributed among the genotypes.

Conclusions: Genetic correlation between three genetic components revealed that both additive and epistasis play a great role among some studied traits suggesting common genetic pool. Thus, selection based on additive gene action based on indirect selection could improve cotton yield. The genotypes which have large genetic diversity could produce significant general or specific combining ability which may be reflecting its genetic behavior.

\section{Introduction}

Cotton is the most important commercial fibre crop playing key role in economic, political and social development of the world. The Egyptian cotton breeding program (Gossypium barbadense L.,) is depending on producing pure lines to release new varieties. The main emphasis of breeding program is to develop new varieties with high yield potential and superior fiber quality traits. Since genetic improvement process never ends. Cotton yield is a polygenic trait and based on its components (El-Mansy et al., 2014). Cotton yield components may link or segregate independently, so the cotton breeder should investigate the genetic control and behavior for yield, its components and fiber quality traits.

The success of any breeding program based on the available data about genetic variation in the studied population. So, the plant breeders used many biometrical techniques to estimate or measure genetic variation and classified to its components. The available results or data provide genetic information about the inheritance of the economic quantitative traits (Jayade et al., 2014). Finally, the plant breeders know how to manage and lead breeding program. Fisher was the first one classified genetic variation into additive (D), dominance (H) and epistasis (i) components (Sing and Narayanan, 2013).

Also, understand combining ability effects is essential for efficient breeding program. Combining ability define as the estimation of the genotypes value on the basis of their offspring performance in some definite mating designs. General combining ability (GCA) is associated with additive gene effects; while specific combining ability (SCA) is related to dominance and epistatic effects if it is present (Fasahat et al 2016). The GCA of a line can be used in plant breeding as a measure of its breeding value. Also, GCA considered a good indicator for the relative value of the parental lines or frequency of favorable alleles and its genetic divergence, which help to select the superior parental lines. The differences between pure lines in GCA are due to the additive or additive $\times$ additive gene interactions (if epistasis is present), while the differences in the SCA of combinations are related to non-additive, often dominant epistatic interactions (Sing and Narayanan, 2013).

Triple test cross (TTC) designed by Kearsey and Jinks, 1968 which is one of the best matting design for detecting and estimating the fixable and non-fixable genetic components for quantitative traits. Therefore, classified epistasis to its components (additive $\times$ additive and additive $\times$ dominance), which should be studied precisely before starting any breeding program. The output analysis could help plant breeder to choose the best breeding or selection procedures to be followed for breeding program improvement. Also, provide more knowledge about genetic correlation between the three genetic components (additive, dominance and epistasis), which occurs between traits, that can help breeder to increase selection efficiency by using favorable trait combinations to minimize the retarding effect of negative correlations between different traits. So many Egyptian cotton breeders used TTC analysis to understand the genetic basis of cotton yield and fiber quality traits Soliman et al., 2008; El-Lawendey et al., 2010; Saleh 2013 and Mahros, 2016.

Genetic diversity is the foundation to release new cotton varieties. It is essential to the plant breeder to detect and know more information about genetic diversity and select the more diverse parents for hybridization breeding program to increase transgreesive segregation. The accumulation knowledge about genetic diversity and genetic inheritance or control of the quantitative traits could help plant breeder to increase

Page $2 / 25$ 
population variability combined with selection to favorable alleles. So, many Egyptian cotton breeder study genetic diversity among Egyptian cotton genotypes Abd El-Moghny et al., 2015 a and b.

The aim of the study is to create $26\left(13 \mathrm{G}_{\mathrm{i}} \times \mathrm{L}_{1}\right.$ and $\left.13 \mathrm{G}_{\mathrm{i}} \times \mathrm{L}_{2}\right)$ cotton crosses using 13 cotton lines $\left(\mathrm{G}_{\mathrm{i}}\right)$ and two testers $\left(\mathrm{L}_{1}\right.$ and $\left.\mathrm{L}_{2}\right)$ to obtain useful information about the relationship between genetic component (fixable and non-fixable), combining ability and genetic diversity. Also, the study will extend to estimate genetic correlations between genetic components by using modified triple test cross analysis for twelve quantitative cotton traits.

\section{Materials And Methods}

The genetic materials used in the present investigation were involved fifteen cotton genotypes derived from three origins belong to Gossypium barbadense L. Origin, pedigree and category of these genotypes are presented in Table 1. The selfed seeds of these genotypes were kindly supported from the Cotton Breeding and Cotton Maintance Departments; Cotton Research Institute, Agriculture Research Center, Giza, Egypt. The present investigation was carried out at Sakha Experimental Station; Agriculture Research Center, Kafr El-Sheikh government; Egypt, during the three growing seasons of 2017-2019.

The selfed seeds of the fifteen cotton genotypes were evaluated for twelve quantitative yield and fiber quality traits during the growing seasons 2017 to obtain more information about the highest and lowest performing cotton genotypes to be used as testers to construct modified triple test cross (TTC) population. The experimental design was randomized complete blocks design (RCBD) with three replications. Each replicate consists of four rows for each genotype.

In the growing season of 2018, each of the thirteen cotton genotypes $\left(G_{i}=1\right.$ to 13$)$ were crossed to the two testers Alexandra 4 ; $\left(L_{1}\right)$ and Bahtim $\left(\mathrm{L}_{2}\right)$ to produce $26 \mathrm{~F}_{1}$ crosses; consists of $13 \mathrm{~L}_{1}\left(\mathrm{G}_{\mathrm{i}} \times \mathrm{L}_{1}\right)$ and $13 \mathrm{~L}_{2}\left(\mathrm{G}_{\mathrm{i}} \times \mathrm{L}_{2}\right)$, respectively where $\mathrm{G}_{\mathrm{i}}=1$ to 13 .

In the growing season of 2019 , the seeds of 26 families $\left(13 \mathrm{~L}_{1}+13 \mathrm{~L}_{2}\right)$ with their fifteen parental genotypes were sown in a randomized complete blocks design (RCBD) with three replications each replicate was consists of four rows for both parents and their $F_{1}$ crosses. Row was $4.0 \mathrm{~m}$ long; the distance between rows $0.7 \mathrm{~m}$ and within plants $0.4 \mathrm{~m}$ to insure 10 plants per row. Hills were thinned to keep a constant stand of one plant per hill at seedlings stage. All agronomic cultural practices were applied as usually recommended for ordinary cotton fields.

At harvest the inner eight individual plants of the fifteen parental cotton genotypes with their derived 26 hybrids were harvested from each row and ginned in order to, estimate six agronomic traits. The data were recorded on boll weight (BW) in grams as the average weight of ten bolls per plant, seed cotton yield per plant (SCY) in grams, lint yield per plant (LY) in grams, lint percentage (L\%), seed index (SI) and lint index (LI) in grams. Also, six fiber quality traits; fiber length (FL), fiber strength (FS), fiber fineness (FF), uniformity ratio (UR\%) yellowness (+ b) and fiber reflectance $(\mathrm{Rd})$ were estimated at Cotton Technology Laboratory, Cotton Research Institute, Agricultural Research Center, Giza, Egypt. 
Table 1

Origin, pedigree and category for the fifteen parental cotton genotypes

\begin{tabular}{|c|c|c|c|c|}
\hline No. & Parents & Origin & Pedigree & Category \\
\hline \multicolumn{5}{|c|}{ Lines $\left(G_{i}\right)$} \\
\hline$P_{1}$ & Karshenky & Russia & Unknown & Long staple \\
\hline$P_{2}$ & Suvin & India & Sujata $\mathrm{x}$ Vincent & Long staple \\
\hline$P_{3}$ & Ashmouni & Egypt & Ashmouni & Long staple \\
\hline $\mathbf{P}_{4}$ & Giza 45 & Egypt & Giza 28 x Giza 7 & Extra long staple \\
\hline$P_{5}$ & Giza 70 & Egypt & Giza 59A x Giza 51B & Extra long staple \\
\hline$P_{6}$ & Giza 76 & Egypt & Menoufi x Pima & Extra long staple \\
\hline$P_{7}$ & Giza 77 & Egypt & Giza 70 x Giza 68 & Extra long staple \\
\hline $\mathbf{P}_{8}$ & Giza 80 & Egypt & Giza 66 x Giza 73 & Long staple \\
\hline$P_{9}$ & Giza 81 & Egypt & Giza $67 \times 5844 A$ & Long staple \\
\hline$P_{10}$ & Giza 88 & Egypt & (Giza 77 x Giza 45) B & Extra long staple \\
\hline$P_{11}$ & Giza 89 & Egypt & Giza $75 \times 6022$ & Long staple \\
\hline$P_{12}$ & Giza 89 x S6 & Egypt & $(($ Giza $75 \times 6022) \times$ S6) & Long staple \\
\hline$P_{13}$ & Giza 86 & Egypt & Giza 75 x Giza 81 & Long staple \\
\hline \multicolumn{5}{|c|}{ Testers $\left(L_{1}\right.$ and $\left.L_{2}\right)$} \\
\hline $\mathrm{L}_{1}$ & Alexandria 4 & Egypt & Unknown & Long staple \\
\hline $\mathrm{L}_{2}$ & Bahtim & Egypt & Unknown & Long staple \\
\hline
\end{tabular}

\section{Biometrical analysis:}

Before proceeding to modified triple test cross analysis, the recorded data were subjected to analysis of variance according to Gomez and Gomez, 1984 to determine significant differences among genotypes. The variation among parental cotton genotypes $\left(\mathrm{G}_{\mathrm{i}}\right)$ and their crosses $\left(\mathrm{L}_{1}\right)$ and $\left(\mathrm{L}_{2}\right)$ was partitioned into two sources to general combining ability (GCA) and specific combining ability (SCA) effects were calculated in accordance with the procedure suggested by Kempthorne, 1957.

The detection of epistasis was done according to the method outlined by Jinks et al., 1969 and based on the genetic model;

$L i j k=M+G i j+R k+E i j k$

Where,

$\mathrm{L}_{\mathrm{ijk}}=$ Phenotypic value of cross between tester $\mathrm{i}$ and line $\mathrm{j}$ in $\mathrm{k}$ replication .

$M=$ Overall mean of all single and three way crosses.

$\mathrm{G}_{\mathrm{ij}}=$ Genotypic value of cross between tester $\mathrm{i}$ and line $\mathrm{j}$.

$\mathrm{R}_{\mathrm{k}}=$ Effect of $\mathrm{k}^{\text {th }}$ replication .

$E_{i j k}=$ Error.

The treatment means were subjected to analysis of variance according to the modified TTC method (Ketata et al., 1976) as illustrated by Sharma, 1988 and Khattak et al., 2002. The analysis of variance of $\left(L_{1}, L_{2}, G_{i}\right)$ and $\left(L_{1}, L_{2}\right)$ sets of families have been carried out for every trait separately. This method tests epistasis based on the variance between $\left(L_{1 i}+L_{2 i}-G_{i}\right)$ for $i=1,2 \ldots 13$, where $L_{1}$ is the mean of the cross of the $i^{\text {th }}$ 
line with tester $L_{1}$ and $L_{2}$, respectively. The crosses mean of the $i^{\text {th }}$ line with tester $L_{2}$; and $G_{i}$ is the mean of the $i^{\text {th }}$ line. While, the within $\left(L_{1 i}\right.$ and $\left.L_{2 i}\right)$ types of families are appropriate for testing the significance of additive $\left(L_{1 i}+L_{2} i\right)$ and dominance $\left(L_{1 i}-L_{2 i}\right)$ gene effects. For test epistasis significant thirteen values of $\left(L_{1 i}+L_{2 i}-G_{i}\right), G_{i}=1$ to 13 with 12 degrees of freedom was used to test overall epistasis. The overall epistasis was partitioned into (i) type of epistasis (additive $x$ additive) and $(i+j)$ type (additive $x$ dominance) gene interactions (Jinks and Virk, 1977). Also, the mean squares due to sums $\left(L_{1 i}+L_{2 i}\right)$ and differences $\left(L_{1} i-L_{2 i}\right)$ for 12 degrees of freedom were used to detect significant of both additive and dominance gene effects.

The direction of dominance $(F)$ was computed from covariance of sums $\left(L_{1 i}+L_{2 i}\right) /$ differences $\left(L_{1 i}-L_{2 i}\right)$ which equal $=-1 / 8$. The correlation coefficient of sums / differences was used to test significance of F-value. The estimation of additive (D), dominance (H) and epistasis genetic components were obtained to detect the direction of dominance. The degree of dominance was calculated as (H/D) ${ }^{1 / 2}$. Genetic correlation for genetic components was calculated between additive $\left(r_{D}\right)$, dominance $\left(r_{H}\right)$ and epistasis $\left(r_{i}\right)$. Correlation coefficients were computed from $\left(L_{1 i}+\right.$ $\left.L_{2 i}\right),\left(L_{1 i}-L_{2 i}\right)$ and $\left(L_{1 i}+L_{2 i}-G_{i}\right)$, respectively.

Cluster analysis using multivariate analysis based on yield, its components and fiber quality traits using averaged linkage (within groups) was calculated as outlined by Johnson and Wichern 1998 to investigate genetic dissimilarity between fifteen parental cotton genotypes. The dissimilarity coefficient and dendrogram were done by using SPSS software.

\section{Results And Discussion}

The improvement of any breeding program depending on how much information is available to the plant breeders about the crop and their target traits. Hybridization is an important source to induce genetic variability, so cotton breeders use different mating models to pass favorable alleles to the next progeny. The plant materials used in this study consists of fifteen cotton genotypes belonging to Gossypium barbadense L., and their twenty six cotton crosses. Analysis of variance (ANOVA) for all the agronomic and fiber quality traits was used to detect the variability between genotypes (Table 2) which revealed highly significant differences for genotypes, crosses, parents, lines and testers for all the studied traits indicating the presence of considerable variability among these genotypes and showed existence worth of variations between the two testers $\left(L_{1}\right.$ and $\left.L_{2}\right)$ which resulted into the expression of high mean performance of their $F_{1}$ crosses. Also, provide precise estimates of additive and dominance variance as reported by Kearsey and Jinks 1968. However, line vs. tester showed highly significant differences for all the studied traits except uniformity ratio, yellowness and fiber reflectance, showing the importance of both additive and nonadditive variance gene effects. The same results for different cotton crosses belonging to Gossypium barbadense L., obtained by AL-Hibbiny, 2015; Mahrous, 2018; Sultan et al., 2018 and Yehia and El-Hashash, 2019. These results could be adequate for proceeding a further biometrical analysis by means using modified triple test cross (TCC).

The phenotypic mean values of backcrosses $L_{1}, L_{2}$ and parents $\left(G_{i}\right)$ exhibited significant differences for most studied traits (Tables 3 and 4 ). Generally, backcrosses to the second tester (Bahtim) showed higher mean values for six yield traits. Such values were varied from line to other, the backcross of the two testers with Giza 86 surpassed all the other genotypes for all yield traits followed by the Indian genotype (Suvin) and the Egyptian variety Giza 80 . These results might reflect the conspicuous genetic constitution of the Egyptian varieties Giza 86 and Giza 80 as well as the Indian one which might possess much potential to improve yield traits.

The reverse trend was observed for fiber quality traits since backcrosses with $\mathrm{L}_{1}$ tended to improve most fiber quality traits, which showed decrease values of micronaire reading (desirable values) and increased fiber length, fiber strength and uniformity ratio. The Egyptian lines Giza 45 and Giza 77 surpassed other lines when used as common parents in TTC and recorded desirable fiber quality values over the two testers. Similar conclusions were reported by El-Mansy et al., 2010 and 2012.

Generally, the twenty six cotton crosses showed a wide range of variability for all the studied traits represent two categories for fiber quality long and extra-long staple category. The two yield traits; seed cotton yield and lint yield for the $13\left(G_{i} \times L_{2}\right)$ was larger than the other $13\left(G_{i} \times L_{1}\right)$ among all crosses. 
Table 2

Analysis of variance for the triple test crosses for all the studied traits

\section{Mean Squares}

Yield and its components

\begin{tabular}{|c|c|c|c|c|c|c|c|}
\hline S.O.V & d.f & $\begin{array}{l}\text { Boll weight } \\
\text { g }\end{array}$ & $\begin{array}{l}\text { Seed cotton } \\
\text { yield } \\
\text { g }\end{array}$ & $\begin{array}{l}\text { Lint yield } \\
\text { g }\end{array}$ & Lint \% & $\begin{array}{l}\text { Seed index } \\
\text { g }\end{array}$ & $\begin{array}{l}\text { Lint inde) } \\
\text { g }\end{array}$ \\
\hline Genotypes & 40 & $0.244^{\star \star}$ & $1380.051^{\star \star}$ & $203.240 * *$ & 11.331 ** & $1.509 * \star$ & $1.440 * *$ \\
\hline
\end{tabular}

$\left(G_{i}\right)+\left(G i x L_{1}\right)+(G i x$

$\mathrm{L}_{2}$ )

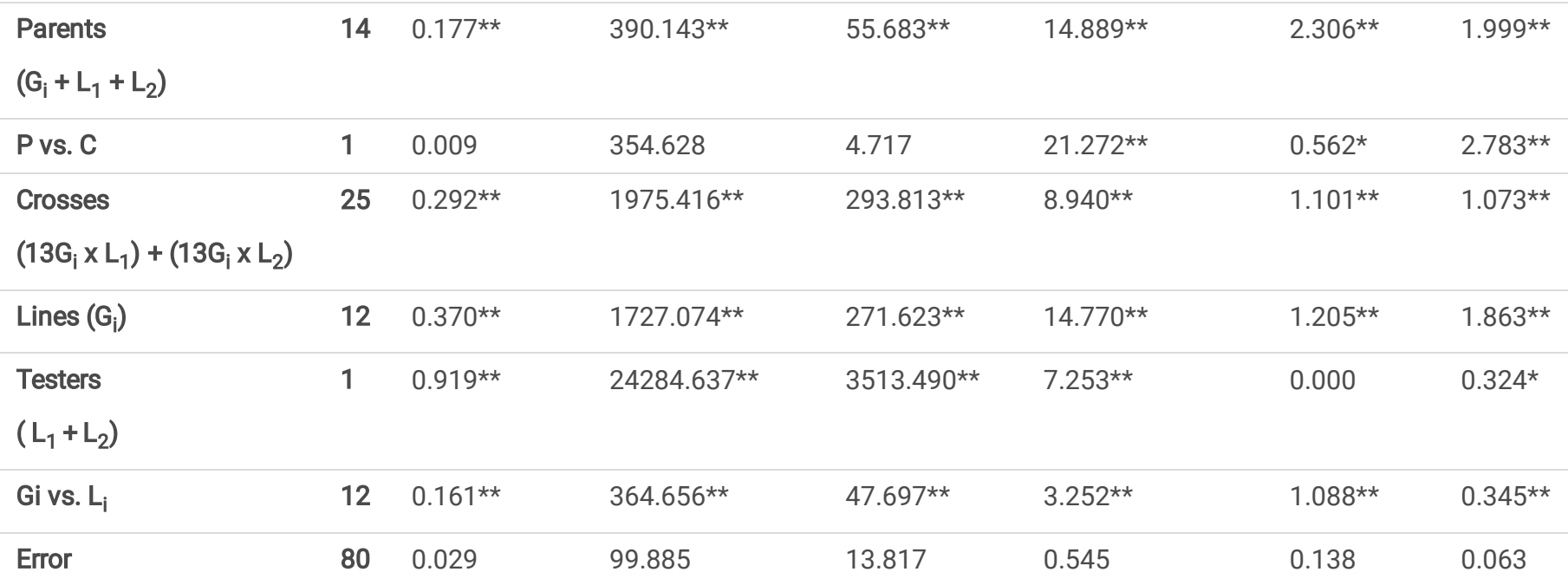

Fiber quality traits

\begin{tabular}{|c|c|c|c|c|c|c|c|}
\hline sov & d.f & $\begin{array}{l}\text { Fiber } \\
\text { fineness }\end{array}$ & $\begin{array}{l}\text { Fiber length } \\
\text { mm }\end{array}$ & $\begin{array}{l}\text { Fiber } \\
\text { strength }\end{array}$ & $\begin{array}{l}\text { Uniformity ratio } \\
\%\end{array}$ & Yellowness & $\begin{array}{l}\text { Fiber } \\
\text { reflectance }\end{array}$ \\
\hline Replications & 2 & 0.018 & 0.188 & 0.186 & 0.018 & 0.270 & 2.218 \\
\hline $\begin{array}{l}\text { Genotypes } \\
\left(G_{i}\right)+\left(G i \times L_{1}\right) \\
\left.L_{2}\right)\end{array}$ & 40 & $0.431^{\star \star}$ & $0.807 \star \star$ & $7.241^{\star \star}$ & $0.431^{\star *}$ & $4.080 \star \star$ & $22.886^{\star \star}$ \\
\hline $\begin{array}{l}\text { Parents } \\
\left(G_{i}+L_{1}+L_{2}\right)\end{array}$ & 14 & $0.367 * \star$ & $0.821^{\star \star}$ & $9.214^{\star \star}$ & $0.367 * \star$ & $6.307^{\star \star}$ & 32.038 ** \\
\hline P vs. C & 1 & $0.777 * \star$ & $0.470^{\star}$ & 13.580 ** & 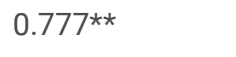 & $3.264^{\star \star}$ & 21.893 \\
\hline $\begin{array}{l}\text { Crosses } \\
\left(13 G_{i} \times L_{1}\right)+(\end{array}$ & 25 & $0.453^{\star \star}$ & $0.813^{\star \star}$ & $5.883^{\star \star}$ & $0.453 *$ & $2.865^{\star \star}$ & $17.800^{\star \star}$ \\
\hline Lines $\left(G_{i}\right)$ & 12 & $0.478 * \star$ & $0.783^{\star \star}$ & $7.504^{\star \star}$ & $0.478 * \star$ & $5.220 * \star$ & $33.587 * \star$ \\
\hline $\begin{array}{l}\text { Testers } \\
\left(L_{1}+L_{2}\right)\end{array}$ & 1 & $1.468 * \star$ & $0.525^{\star}$ & 0.003 & 1.468 & $6.606^{\star *}$ & 0.431 \\
\hline Gi vs. $L_{i}$ & 12 & $0.344^{\star \star}$ & $0.868 * \star$ & $4.752^{\star \star}$ & 0.344 & 0.199 & 3.461 \\
\hline Error & 80 & 0.058 & 0.120 & 1.051 & 0.058 & 0.185 & 4.616 \\
\hline
\end{tabular}


Table 3

Phenotypic mean performance for thirteen cotton genotypes $\left(G_{i}\right)$, tester one $\left(L_{1}\right)$ with their $13\left(G_{i} \times L_{1}\right)$ and tester two $\left(L_{2}\right)$ with their $13\left(G_{i} \times L_{2}\right)$ crosses for the studied yield and its component traits

\begin{tabular}{|c|c|c|c|c|c|c|c|c|c|}
\hline \multirow[t]{4}{*}{ Genotypes } & \multicolumn{3}{|c|}{ Boll weight } & \multicolumn{3}{|c|}{ Seed cotton yield } & \multicolumn{3}{|c|}{ Lint yield } \\
\hline & \multicolumn{3}{|l|}{ BW } & \multicolumn{3}{|l|}{ SCY } & \multicolumn{3}{|l|}{ LY } \\
\hline & \multicolumn{3}{|l|}{ g } & \multicolumn{3}{|l|}{ g } & \multicolumn{3}{|l|}{ g } \\
\hline & $\mathbf{P}$ & $\left(G_{i} \times L_{1}\right)$ & $\left(G i x L_{2}\right)$ & $P$ & $\left(G_{i} \times L_{1}\right)$ & $\left(G_{i} \times L_{2}\right)$ & $\mathbf{P}$ & $\left(G_{i} \times L_{1}\right)$ & $\left(G_{i} \times L_{2}\right)$ \\
\hline Karshenky & 2.600 & 2.567 & 2.411 & 92.07 & 61.43 & 84.97 & 31.00 & 20.30 & 30.23 \\
\hline Suvin & 3.078 & 2.500 & 3.267 & 106.17 & 63.80 & 107.97 & 37.07 & 23.67 & 37.87 \\
\hline Ashmouni & 2.611 & 2.433 & 2.789 & 80.27 & 49.97 & 64.27 & 29.97 & 19.00 & 25.27 \\
\hline Giza 45 & 2.622 & 2.078 & 2.556 & 88.27 & 43.47 & 84.57 & 29.13 & 14.63 & 29.20 \\
\hline Giza 70 & 2.278 & 2.722 & 2.911 & 79.93 & 49.07 & 110.80 & 27.43 & 17.63 & 41.47 \\
\hline Giza 76 & 2.667 & 2.833 & 2.933 & 81.50 & 67.60 & 99.53 & 27.37 & 24.37 & 36.13 \\
\hline Giza 77 & 2.956 & 2.978 & 2.656 & 85.80 & 93.73 & 119.70 & 30.80 & 35.40 & 45.50 \\
\hline Giza 80 & 3.033 & 2.956 & 3.078 & 66.03 & 57.53 & 86.83 & 26.67 & 21.77 & 34.93 \\
\hline Giza 81 & 3.178 & 3.200 & 3.144 & 76.13 & 69.80 & 81.87 & 28.97 & 26.17 & 31.40 \\
\hline Giza 88 & 3.011 & 2.833 & 3.356 & 107.23 & 68.37 & 119.93 & 39.90 & 23.77 & 44.90 \\
\hline Giza 89 & 2.678 & 2.522 & 2.911 & 90.50 & 89.17 & 142.17 & 33.43 & 34.27 & 53.53 \\
\hline Giza 89 x S6 & 2.789 & 2.467 & 3.033 & 85.57 & 67.93 & 91.27 & 31.47 & 25.70 & 34.23 \\
\hline Giza 86 & 3.044 & 3.156 & 3.022 & 101.97 & 79.27 & 126.03 & 40.20 & 30.93 & 47.43 \\
\hline Alexandria $4\left(L_{1}\right)$ & 2.722 & & & 78.27 & & & 28.97 & & \\
\hline Bahtim $\left(L_{2}\right)$ & 2.756 & & & 91.47 & & & 30.87 & & \\
\hline Mean & 2.80 & 2.71 & 2.93 & 87.41 & 66.24 & 101.53 & 31.55 & 24.43 & 37.85 \\
\hline Overall mean & 2.81 & & & 85.18 & & & 31.29 & & \\
\hline LSD 0.05 & 0.231 & & & 13.58 & & & 5.05 & & \\
\hline LSD 0.01 & 0.329 & & & 19.37 & & & 7.21 & & \\
\hline \multirow[t]{4}{*}{ Genotypes } & \multicolumn{3}{|c|}{ Lint percentage } & \multicolumn{3}{|c|}{ Seed index } & \multicolumn{3}{|c|}{ Lint index } \\
\hline & \multicolumn{3}{|c|}{$L \%$} & \multicolumn{3}{|l|}{ SI } & \multicolumn{3}{|l|}{ LI } \\
\hline & & & & g & & & g & & \\
\hline & $\mathbf{P}$ & $\left(G_{i} \times L_{1}\right)$ & $\left(G i \times L_{2}\right)$ & $\mathbf{P}$ & $\left(G_{i} \times L_{1}\right)$ & $\left(G_{i} \times L_{2}\right)$ & $\mathbf{P}$ & $\left(G_{i} \times L_{1}\right)$ & $\left(G_{i} \times L_{2}\right)$ \\
\hline Karshenky & 33.724 & 33.171 & 35.587 & 9.800 & 9.533 & 9.733 & 4.989 & 4.739 & 5.376 \\
\hline Suvin & 34.855 & 37.072 & 35.103 & 11.133 & 9.133 & 11.333 & 5.958 & 5.379 & 6.129 \\
\hline Ashmouni & 37.232 & 38.117 & 39.279 & 10.333 & 10.133 & 10.000 & 6.130 & 6.243 & 6.470 \\
\hline Giza 45 & 33.018 & 33.758 & 34.534 & 11.133 & 8.333 & 9.600 & 5.488 & 4.245 & 5.064 \\
\hline Giza 70 & 34.383 & 35.928 & 37.407 & 8.467 & 10.467 & 10.200 & 4.432 & 5.868 & 6.096 \\
\hline Giza 76 & 33.499 & 36.003 & 36.312 & 8.600 & 10.133 & 9.600 & 4.337 & 5.696 & 5.473 \\
\hline Giza 77 & 35.916 & 37.781 & 37.970 & 8.667 & 10.733 & 9.867 & 4.859 & 6.518 & 6.040 \\
\hline Giza 80 & 40.306 & 37.833 & 40.236 & 10.333 & 10.133 & 9.433 & 6.981 & 6.167 & 6.352 \\
\hline Giza 81 & 38.038 & 37.482 & 38.332 & 10.700 & 10.400 & 9.867 & 6.568 & 6.235 & 6.138 \\
\hline Giza 88 & 37.204 & 34.649 & 37.440 & 10.467 & 10.000 & 10.067 & 6.201 & 5.310 & 6.026 \\
\hline
\end{tabular}




\begin{tabular}{|c|c|c|c|c|c|c|c|c|c|}
\hline \multirow[t]{4}{*}{ Genotypes } & \multicolumn{3}{|c|}{ Boll weight } & \multicolumn{3}{|c|}{ Seed cotton yield } & \multicolumn{3}{|c|}{ Lint yield } \\
\hline & \multicolumn{3}{|l|}{ BW } & \multicolumn{3}{|l|}{ SCY } & \multicolumn{3}{|l|}{ LY } \\
\hline & \multicolumn{3}{|l|}{ g } & \multicolumn{3}{|l|}{ g } & \multicolumn{3}{|l|}{ g } \\
\hline & $\mathbf{P}$ & $\left(G_{i} \times L_{1}\right)$ & $\left(G i \times L_{2}\right)$ & $\mathbf{P}$ & $\left(G_{i} \times L_{1}\right)$ & $\left(G_{i} \times L_{2}\right)$ & $\mathbf{P}$ & $\left(G_{i} \times L_{1}\right)$ & $\left(G_{i} \times L_{2}\right)$ \\
\hline Giza 89 & 36.895 & 38.417 & 37.665 & 9.000 & 9.633 & 9.467 & 5.260 & 6.008 & 5.720 \\
\hline Giza 89 x S6 & 36.800 & 37.869 & 37.517 & 10.200 & 10.733 & 10.200 & 5.941 & 6.543 & 6.126 \\
\hline Giza 86 & 39.461 & 39.021 & 37.647 & 9.933 & 10.667 & 10.667 & 6.474 & 6.818 & 6.438 \\
\hline Alexandria $4\left(\mathrm{~L}_{1}\right)$ & 37.087 & & & 9.467 & & & 5.379 & & \\
\hline Bahtim $\left(L_{2}\right)$ & 33.706 & & & 9.700 & & & 4.714 & & \\
\hline Mean & 36.14 & 36.70 & 37.31 & 9.81 & 10.00 & 10.00 & 5.58 & 5.83 & 5.96 \\
\hline Overall mean & 36.31 & & & 9.93 & & & 5.78 & & \\
\hline LSD at 0.05 & 1.003 & & & 0.523 & & & 0.340 & & \\
\hline LSD at 0.01 & 1.431 & & & 0.746 & & & 0.486 & & \\
\hline
\end{tabular}


Table 4

Phenotypic mean performance for thirteen cotton genotypes $\left(G_{i}\right)$, tester one $\left(L_{1}\right)$ with their $13\left(G_{i} \times L_{1}\right)$ and tester two $\left(L_{2}\right)$ with their $13\left(G_{i} \times L_{2}\right)$ crosses for all the studied fiber quality traits

\begin{tabular}{|c|c|c|c|c|c|c|c|c|c|}
\hline \multirow[t]{3}{*}{ Genotypes } & \multirow{2}{*}{\multicolumn{3}{|c|}{$\begin{array}{l}\text { Fiber fineness } \\
\text { FF }\end{array}$}} & \multirow{2}{*}{\multicolumn{2}{|c|}{$\begin{array}{l}\text { Fiber length } \\
\text { FL } \\
\mathrm{mm}\end{array}$}} & & \multirow{2}{*}{\multicolumn{3}{|c|}{$\begin{array}{l}\text { Fiber strength } \\
\text { FS }\end{array}$}} \\
\hline & & & & & & & & & \\
\hline & $\mathbf{P}$ & $\left(G_{i} x L_{1}\right)$ & $\left(G i \times L_{2}\right)$ & \multicolumn{2}{|r|}{$\left(G_{i} \times L_{1}\right)$} & $\left(G_{i} \times L_{2}\right)$ & $\mathbf{P}$ & $\left(G_{i} \times L_{1}\right)$ & $\left(G_{i} \times L_{2}\right)$ \\
\hline Karshenky & 3.4 & 3.1 & 3.2 & 31.267 & 32.633 & 32.200 & 9.633 & 9.833 & 9.367 \\
\hline Suvin & 3.8 & 3.3 & 4.1 & 31.467 & 32.833 & 31.600 & 9.000 & 9.100 & 10.233 \\
\hline Ashmouni & 3.8 & 3.9 & 4.1 & 31.067 & 32.300 & 30.867 & 9.500 & 9.533 & 9.533 \\
\hline Giza 45 & 3.1 & 3.5 & 3.3 & 34.900 & 34.033 & 34.633 & 10.633 & 10.500 & 10.000 \\
\hline Giza 70 & 3.6 & 3.5 & 4.4 & 34.900 & 35.233 & 34.433 & 9.333 & 10.567 & 10.100 \\
\hline Giza 76 & 3.1 & 4.1 & 3.6 & 34.467 & 35.833 & 35.600 & 9.100 & 11.033 & 9.200 \\
\hline Giza 77 & 3.0 & 3.4 & 4.1 & 34.700 & 35.433 & 34.800 & 10.233 & 10.467 & 9.567 \\
\hline Giza 80 & 4.0 & 3.9 & 3.6 & 31.000 & 31.933 & 34.867 & 9.567 & 10.267 & 9.700 \\
\hline Giza 81 & 3.5 & 4.1 & 4.1 & 31.700 & 34.633 & 32.900 & 9.800 & 9.600 & 9.700 \\
\hline Giza 88 & 3.8 & 3.4 & 4.0 & 34.600 & 32.433 & 35.200 & 9.867 & 9.300 & 9.633 \\
\hline Giza 89 & 3.9 & 3.7 & 4.0 & 30.033 & 33.533 & 32.533 & 9.300 & 9.333 & 9.300 \\
\hline Giza 89 x S6 & 3.7 & 3.6 & 4.5 & 32.767 & 34.767 & 32.333 & 9.200 & 10.033 & 10.833 \\
\hline Giza 86 & 4.2 & 4.1 & 4.1 & 33.100 & 33.433 & 33.900 & 10.000 & 9.500 & 9.767 \\
\hline Alexandria $4\left(\mathrm{~L}_{1}\right)$ & 4.0 & & & 32.100 & & & 10.733 & & \\
\hline Bahtim $\left(L_{2}\right)$ & 3.6 & & & 32.267 & & & 9.867 & & \\
\hline Mean & 3.63 & 3.66 & 3.93 & 33.08 & 33.77 & 33.76 & 9.71 & 9.93 & 9.76 \\
\hline Overall mean & 3.74 & & & 33.51 & & & 9.80 & & \\
\hline LSD at 0.05 & 0.328 & & & 1.393 & & & 0.471 & & \\
\hline LSD at 0.01 & 0.467 & & & 1.988 & & & 0.672 & & \\
\hline \multirow[t]{3}{*}{ Genotypes } & \multicolumn{3}{|c|}{ Uniformity ratio } & \multicolumn{3}{|c|}{ Yellowness } & \multicolumn{3}{|c|}{ Fiber reflectance } \\
\hline & \multicolumn{3}{|l|}{ UR\% } & \multicolumn{3}{|l|}{$+b$} & \multicolumn{3}{|l|}{ Rd } \\
\hline & $\mathbf{P}$ & $\left(G_{i} \times L_{1}\right)$ & $\left(G i \times L_{2}\right)$ & $P$ & $\left(G_{i} \times L_{1}\right)$ & $\left(G_{i} \times L_{2}\right)$ & $\mathbf{P}$ & $\left(G_{i} \times L_{1}\right)$ & $\left(G_{i} \times L_{2}\right)$ \\
\hline Karshenky & 94.3 & 91.5 & 92.2 & 10.167 & 10.667 & 10.067 & 71.6 & 67.367 & 67.533 \\
\hline Suvin & 89.9 & 89.2 & 90.3 & 9.133 & 10.300 & 10.267 & 72.3 & 70.233 & 68.700 \\
\hline Ashmouni & 87.9 & 90.2 & 88.0 & 9.367 & 10.200 & 9.600 & 74.2 & 70.667 & 70.200 \\
\hline Giza 45 & 86.8 & 90.0 & 91.4 & 9.567 & 9.833 & 9.033 & 73.0 & 72.267 & 71.467 \\
\hline Giza 70 & 89.9 & 91.4 & 92.2 & 9.133 & 11.100 & 10.433 & 71.3 & 67.933 & 66.200 \\
\hline Giza 76 & 90.9 & 93.8 & 93.8 & 8.767 & 10.000 & 8.867 & 73.2 & 72.333 & 72.167 \\
\hline Giza 77 & 91.3 & 93.3 & 92.9 & 12.667 & 11.467 & 10.567 & 67.0 & 68.233 & 68.000 \\
\hline Giza 80 & 89.0 & 90.8 & 93.2 & 12.567 & 12.300 & 12.233 & 63.6 & 66.367 & 65.167 \\
\hline Giza 81 & 90.3 & 92.1 & 90.8 & 9.467 & 11.033 & 10.500 & 71.6 & 69.467 & 71.333 \\
\hline Giza 88 & 89.7 & 90.7 & 93.2 & 12.100 & 12.600 & 11.667 & 64.8 & 64.567 & 66.600 \\
\hline Giza 89 & 91.3 & 91.2 & 89.2 & 8.400 & 9.767 & 9.333 & 71.5 & 72.767 & 71.900 \\
\hline
\end{tabular}




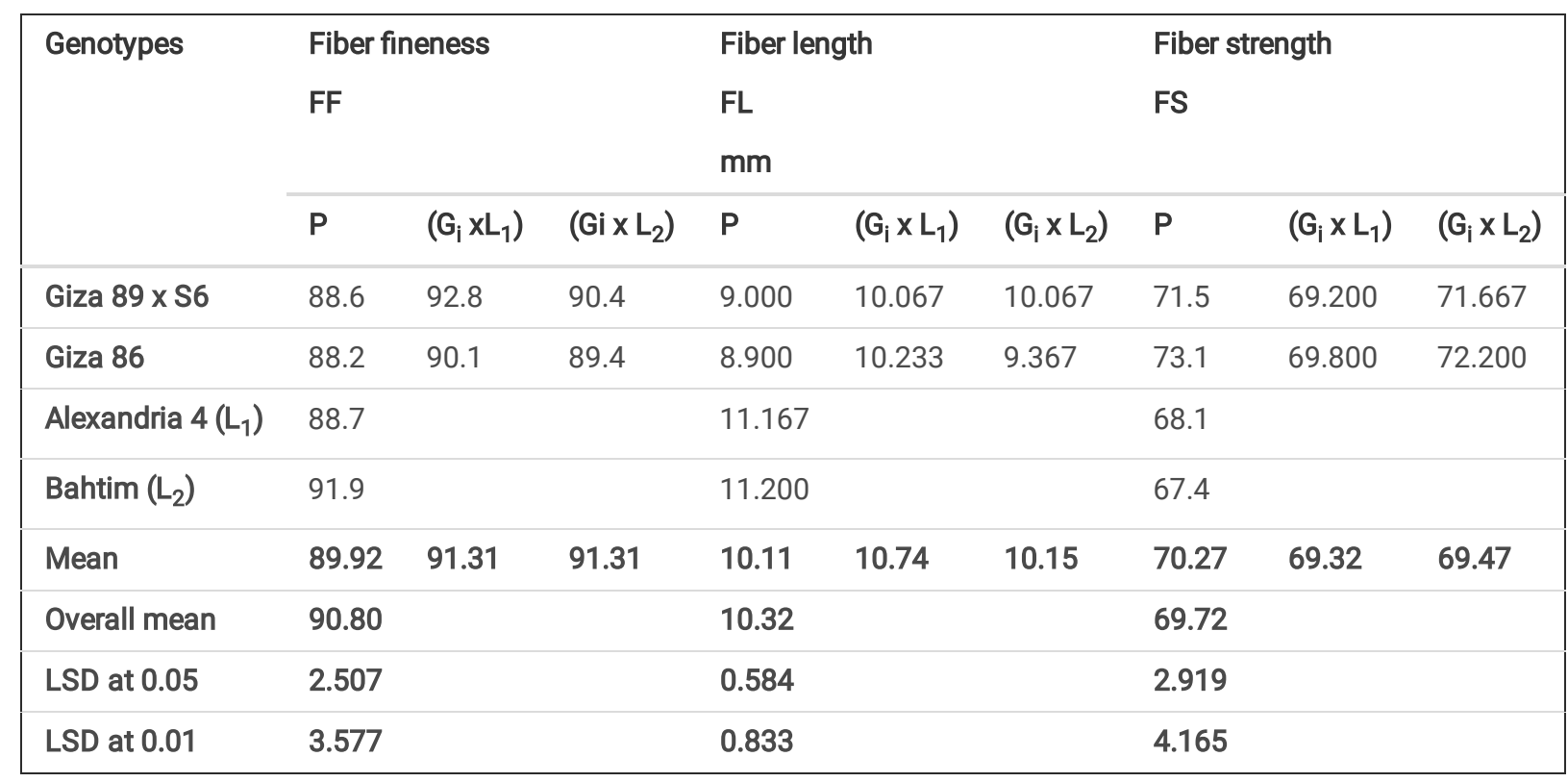

\section{Combining ability (GCA)}

The ultimate to choice parents for any breeding program is generally based on phenotypic performance of parents and their $\mathrm{F}_{1}$ s. However; GCA and SCA effects were more informative than phenotypic performance, since it also revealed the type of gene action, which could help plant breeder to devise breeding or selection strategies.

Estimation of general combining ability (GCA) effects of both 13 lines $\left(G_{i}\right)$ and the two testers $\left(L_{1}\right.$ and $\left.L_{2}\right)$ for all the studied traits is presented in Table 5. The results showed that the two lines Giza 77 and Giza 89 were the best general combiner when recorded significant and positive general combining ability for seed cotton yield, lint yield and lint \% and Giza 88 has the same direction for boll weight, seed cotton yield and lint yield. While, Giza 86 has positive and significant general combining ability (GCA) for all the studied yield traits, so the Egyptian cotton breeders define Giza 86 as a good combiner for yield traits and its components.

On the other hand, tester one Alexandria $4\left(\mathrm{~L}_{1}\right)$ has negative and significant general combining ability for all the studied traits and recorded inferior values for seed index (SI) and lint index ( $\mathrm{LI})$. While, the second tester Bahtim $\left(\mathrm{L}_{2}\right)$ showed the opposed direction for the same traits. These findings may be reflect the higher mean performance for the crosses including second tester $\left(L_{2}\right)$ as a parent than the crosses had first tester $\left(L_{1}\right)$ for seed cotton yield and lint traits. So, the second tester $\left(L_{2}\right)$ has a greater ability to transmit his higher performance to its progeny than tester one $\left(\mathrm{L}_{1}\right)$. For fiber quality traits the parental lines Giza 45 was the best combiner for fiber fineness and fiber strength. The extra-long staple varieties Giza 70, Giza 76 and Giza 77 showed desirable values for fiber length (FL) and uniformity ratio (UR \%). The same results were obtained by many Egyptian breeders Sultan et al., 2018 and Yehia and El-Hashash, 2019 especially for Giza 86 as a good combiner.

Since, general combining ability reflects parental performance and its gene expression; additive and additive $\mathrm{x}$ additive gene type of epistasis if present. The significance of GCA effects for the studied traits showed the importance of fixable gene action. Thus, plant breeder may utilize good or general combiner for specific breeding purposes. The relationship among GCA effects for the studied traits (Table 6) revealed that increasing additive gene effect controlling boll weight was correlated with increasing additive ones for lint \%, seed index, lint index and fiber fineness. Boll weight is the independent key for yield components and played a prime role to managing or increasing yield productivity. Since, yield is one of the most important economic traits in cotton. So, the possibility to improve yield is increasing efficiency of indirect selection for its components (El-Mansy et al., 2014).

The basis of specific combining ability (SCA) effects revealed that most of the combinations having significant SCA effects were related to genetically diverse parents. The major aim for cotton breeding program is to obtain a cross combination with high values for all yield traits. Most of the twenty six cotton cross combination surpassed in both yield and fiber quality traits (Table 7). Two combinations recorded significant positive SCA values for boll weight. The cross (Giza 77 x Alexandria 4) and (Suvin x Bahtim) recorded the best SCA for seed cotton yield and lint yield. While, the two crosses (Giza $80 \times$ Alexandria 4) and (Giza $70 \times$ Bahtim) which include one good and the other poor general combiner could produce desirable transgreesive segregant if complex fixable gene (additive) is present in a good combiner and complementary with epistatic gene effect in the poor combiner and act in the same direction to maximize the desirable attributes. Similar conclusion was reported by El-Mansy et al., 2014 and Sultan et al., 2018. 
The cross (Ashmouni x Alexandria 4) showed the best SCA effects for lint \%. The crosses (Giza 88 x Bahtim) and (Giza 86 x Alexandria 4) recorded significant positive SCA values for lint \% and lint index, respectively. The twenty six cotton crosses did not show superiority for all the studied fiber quality traits. Generally, few combinations showed desirable significant SCA effects for fiber fineness. However, only three and four crosses recorded significant positive values for fiber strength and fiber length, respectively.

Positive and significant correlation was recorded between GCA for all studied yield traits and fiber fineness. Also, the same trend between fiber length with two fiber quality traits; fiber strength and uniformity ratio \% (Table 6). Also, most of yield components traits had positive and significant SCA. While, negative correlation was shown between SCA for fiber fineness and fiber length but significant and positive correlation for SCA between fiber finesses and fiber strength. These results showed that the best specific combinations were not always obtained from parents which showed good or positive general combining ability effects. These findings are inconsistent with those obtained by Ekinci and Basbage, 2015; Yehia and El-Hashash, 2019 and Al-Hibiny et al., 2019.

These results indicated that these genotypes had the capacity to transmit superior performance to its progenies. The GCA is a primarily function of additive genetic variance (D) but if epistasis is present GCA will include additive $x$ additive type (i) of non-allelic interaction, also it's useful to select good parents for hybridization programs. Specific combining ability (SCA) is a function of dominance genetic variance (H), but if epistasis is present it will include two types of non-allelic interaction (additive $x$ dominance and dominance $x$ dominance) and it's useful for selecting superior cross combinations (Sing and Narayanan, 2013 and Fasahat et al., 2016). Therefore, the better understand of combining ability genetic basis will be guidance for cotton improvement programs.

The presence of significance general and specific combining ability effects through $\mathrm{F}_{1}$ generations is a consequence of fluctuations for additive and dominance relationship, respectively among the parents (Basbag et al., 2007). The estimation of additive and dominance genetic components appeared to be biased a result of epistasis lead to kind of discrepancy in the relative importance of genetic components. Thus, the breeder should take epistasis into account in producing genetic models. 
Table 5

Estimates of general combining ability effects for all the studied traits

\begin{tabular}{|c|c|c|c|c|c|c|}
\hline \multicolumn{7}{|c|}{ Yield and its components } \\
\hline \multirow[t]{2}{*}{ Parents } & BW & SCY & LY & L\% & SI & LI \\
\hline & $\mathbf{g}$ & $\mathbf{g}$ & $\mathbf{g}$ & & g & $\mathbf{g}$ \\
\hline \multicolumn{7}{|l|}{ Lines $\left(G_{i}\right)$} \\
\hline Karshenky & -0.331 * & $-10.68 *$ & $-5.876^{\star}$ & $-2.626^{\star}$ & $-0.369 *$ & $-0.835^{\star}$ \\
\hline Suvin & 0.064 & 1.997 & -0.376 & -0.917 * & 0.231 & -0.139 \\
\hline Ashmouni & $-0.21^{\star *}$ & $-26.76^{*}$ & $-9.009 *$ & $1.693^{*}$ & 0.064 & $0.463^{*}$ \\
\hline Giza 45 & -0.503 & $-19.86^{*}$ & $-9.226^{\star}$ & $-2.859 *$ & $-1.036^{\star}$ & $-1.239 *$ \\
\hline Giza 70 & -0.003 & -3.953 & -1.592 & -0.338 & $0.331 *$ & 0.089 \\
\hline Giza 76 & 0.064 & -0.319 & -0.892 & $-0.848 *$ & -0.136 & $-0.308 *$ \\
\hline Giza 77 & -0.003 & $22.831^{*}$ & $9.308 *$ & $0.871 *$ & 0.297 & $0.386^{\star}$ \\
\hline Giza 80 & $0.197 *$ & $-11.70 *$ & -2.792 & 2.030 * & -0.219 & $0.367 *$ \\
\hline Giza 81 & $0.353^{\star \star}$ & $-8.053^{\star}$ & -2.359 & $0.902^{\star}$ & 0.131 & $0.294^{\star}$ \\
\hline Giza 88 & $0.275^{\star \star}$ & $10.264^{*}$ & $3.191 *$ & -0.961 * & 0.031 & $-0.225^{\star}$ \\
\hline Giza 89 & -0.103 & $31.781^{\star}$ & $12.758^{\star}$ & $1.036^{\star}$ & $-0.453^{\star}$ & -0.029 \\
\hline Giza 89 x S6 & -0.070 & -4.286 & -1.176 & $0.688^{*}$ & $0.464^{\star}$ & $0.441^{\star}$ \\
\hline Giza 86 & $0.269 * \star$ & $18.764^{*}$ & $8.041^{*}$ & $1.329 *$ & $0.664 *$ & $0.735^{\star}$ \\
\hline \multicolumn{7}{|c|}{ Testers $\left(L_{1}\right.$ and $\left.L_{2}\right)$} \\
\hline Alexandria 4 & $-0.109 *$ & $-17.65^{\star}$ & $-6.712^{\star}$ & $-0.305^{\star}$ & 0.000 & -0.064 \\
\hline Bahtim & $0.109 *$ & $17.645^{\star}$ & $6.712^{\star}$ & $0.305^{\star}$ & 0.000 & 0.064 \\
\hline \multicolumn{7}{|c|}{ Fiber quality traits } \\
\hline \multirow[t]{2}{*}{ Parents } & $\mathrm{FF}$ & FS & $\mathrm{FL}$ & UR & $+b$ & Rd \\
\hline & & & $\mathrm{mm}$ & $\%$ & & \\
\hline \multicolumn{7}{|l|}{ Lines $\left(G_{i}\right)$} \\
\hline Karshenky & $-0.65^{\star \star}$ & -0.246 & 0.151 & -0.646 & -0.078 & $-1.947 *$ \\
\hline Suvin & -0.079 & -0.179 & -1.549 & $-0.079 \star$ & -0.162 & 0.069 \\
\hline Ashmouni & $0.221 * \star$ & $-0.313^{\star}$ & $-2.182^{\star}$ & $0.221 *$ & $-0.545^{\star}$ & 1.036 \\
\hline Giza 45 & $-0.413^{\star}$ & $0.404^{*}$ & 0.568 & -0.413 & $-1.012^{\star}$ & $2.469 *$ \\
\hline Giza 70 & 0.171 & $0.487 *$ & 1.068 & 0.171 & 0.322 & $-2.331 *$ \\
\hline Giza 76 & 0.054 & 0.271 & 1.951 & $0.054^{\star}$ & $-1.012^{*}$ & $2.853^{\star}$ \\
\hline Giza 77 & -0.063 & 0.171 & 1.351 & $-0.063^{*}$ & $0.572 *$ & -1.281 \\
\hline Giza 80 & -0.029 & 0.137 & -0.365 & -0.029 & $1.822^{\star}$ & -3.631 * \\
\hline Giza 81 & $0.321^{\star}$ & -0.196 & 0.001 & 0.321 & 0.322 & 1.003 \\
\hline Giza 88 & -0.129 & $-0.379 *$ & 0.051 & -0.129 & $1.688^{*}$ & $-3.814^{*}$ \\
\hline Giza 89 & 0.037 & $-0.529 *$ & -0.732 & 0.037 & $-0.895^{\star}$ & $2.936^{\star}$ \\
\hline Giza 89 x S6 & $0.271^{\star \star}$ & $0.587 *$ & -0.215 & 0.271 & -0.378 & 1.036 \\
\hline Giza 86 & $0.287^{\star \star \star}$ & -0.213 & -0.099 & $0.287^{\star}$ & $-0.645^{\star}$ & 1.603 \\
\hline
\end{tabular}

* Significant at $5 \%$ levels of probability. 


\begin{tabular}{|c|c|c|c|c|c|c|}
\hline \multicolumn{7}{|c|}{ Yield and its components } \\
\hline \multicolumn{7}{|c|}{ Testers $\left(\mathrm{L}_{1}\right.$ and $\left.\mathrm{L}_{2}\right)$} \\
\hline Alexandria 4 & $-0.137 *$ & 0.082 & 0.006 & -0.137 & $0.291 *$ & -0.074 \\
\hline Bahtim & $0.137^{\star}$ & -0.082 & -0.006 & 0.137 & $-0.291^{*}$ & 0.074 \\
\hline
\end{tabular}

Table 6

Correlation between general (above diagonal) and specific (below diagonal) combining ability for all the studied traits

\begin{tabular}{|c|c|c|c|c|c|c|c|c|c|c|c|c|}
\hline Traits & $\begin{array}{l}\text { BW } \\
\text { g }\end{array}$ & $\begin{array}{l}\text { SCY } \\
\mathbf{g}\end{array}$ & $\begin{array}{l}\text { LY } \\
\mathbf{g}\end{array}$ & L\% & $\begin{array}{l}\text { SI } \\
\mathbf{g}\end{array}$ & $\begin{array}{l}\text { LI } \\
\mathbf{g}\end{array}$ & $\mathrm{FF}$ & FS & $\begin{array}{l}\mathrm{FL} \\
\mathrm{mm}\end{array}$ & $\begin{array}{l}\text { UR } \\
\%\end{array}$ & $+b$ & Rd \\
\hline BW & & $0.41^{*}$ & $0.47 *$ & $0.54^{*}$ & $0.62^{*}$ & $0.63^{*}$ & 0.61 * & -0.24 & 0.04 & 0.17 & 0.49 & -0.27 \\
\hline SCY & 0.31 & & $0.98 * \star$ & 0.24 & 0.27 & 0.27 & 0.21 & -0.32 & 0.20 & 0.11 & -0.04 & 0.08 \\
\hline LY & 0.25 & $0.97^{\text {** }}$ & & 0.38 & 0.33 & 0.40 & 0.32 & -0.32 & 0.15 & 0.08 & 0.00 & 0.07 \\
\hline L\% & -0.20 & -0.16 & 0.04 & & $0.53^{\star}$ & $0.92^{\star *}$ & $0.77^{\star \star}$ & -0.17 & -0.33 & -0.18 & 0.21 & 0.00 \\
\hline SI & $0.59 * *$ & 0.32 & 0.19 & -0.36 & & $0.811^{\star \star}$ & 0.67 ** & 0.05 & -0.07 & -0.03 & 0.18 & -0.18 \\
\hline LI & $0.46 *$ & 0.21 & 0.23 & $0.39 *$ & 0.71 ** & & $0.83^{\star \star}$ & -0.08 & -0.25 & -0.15 & 0.22 & -0.07 \\
\hline $\mathrm{FF}$ & 0.32 & 0.31 & 0.29 & -0.22 & 0.12 & -0.03 & & 0.05 & -0.16 & -0.17 & -0.07 & 0.26 \\
\hline FS & 0.56 ** & 0.10 & 0.03 & -0.36 & $0.462^{\star}$ & 0.19 & $0.57^{\star \star}$ & & $0.544 *$ & 0.46 & -0.06 & 0.00 \\
\hline $\mathrm{FL}$ & -0.20 & 0.19 & 0.31 & $0.65^{\star \star}$ & 0.01 & 0.47 * & $-0.39 *$ & -0.26 & & $0.85^{\star \star}$ & 0.02 & -0.04 \\
\hline UR\% & 0.09 & 0.40 * & $0.47 *$ & $0.48^{*}$ & 0.32 & $0.67 * \star$ & -0.14 & -0.17 & 0.78 *夫 & & 0.32 & -0.29 \\
\hline$+b$ & $0.45^{\star}$ & -0.16 & -0.19 & -0.20 & 0.21 & 0.06 & 0.32 & $0.62^{\star \star}$ & -0.21 & -0.12 & & -0.90 ** \\
\hline Rd & -0.15 & -0.26 & -0.25 & -0.03 & -0.30 & -0.34 & 0.02 & 0.32 & -0.03 & -0.30 & -0.15 & \\
\hline
\end{tabular}


Table 7

Estimates of specific combining ability effects for $13\left(G_{i} \times L_{1}\right)$ and $13\left(G_{i} \times L_{2}\right)$ cotton crosses among all the studied traits

\begin{tabular}{|c|c|c|c|c|c|c|c|c|c|c|c|c|}
\hline \multirow[t]{2}{*}{ Crosses } & BW & SCY & LY & L\% & SI & LI & $\mathrm{FF}$ & FS & \multirow{2}{*}{$\begin{array}{l}\mathrm{FL} \\
\mathrm{mm}\end{array}$} & \multirow[t]{2}{*}{ UR } & \multirow[t]{2}{*}{$+b$} & \multirow[t]{2}{*}{ Rd } \\
\hline & g & g & g & & g & g & & & & & & \\
\hline$P_{1} \times L_{1}$ & 0.186 & 5.878 & 1.745 & $-0.90 *$ & -0.100 & -0.254 & 0.121 & 0.151 & $-1.29 *$ & 0.121 & 0.009 & -0.01 \\
\hline$P_{2} \times L_{1}$ & -0.28 *ᄎ & -4.438 & -0.388 & $1.289 *$ & $-1.10 *$ & $-0.31^{\star}$ & $-0.279 *$ & $-0.64^{*}$ & 0.610 & -0.27 & -0.27 & 0.841 \\
\hline$P_{3} \times L_{1}$ & -0.069 & 10.495 & 3.578 & -0.276 & 0.067 & -0.049 & 0.054 & -0.082 & 0.710 & 0.054 & 0.009 & 0.308 \\
\hline$P_{4} \times L_{1}$ & -0.130 & -2.905 & -0.572 & -0.083 & $-0.63^{*}$ & $-0.34^{\star}$ & 0.254 & 0.168 & -0.306 & 0.254 & 0.109 & 0.474 \\
\hline$P_{5} \times L_{1}$ & 0.014 & $-13.22^{\star}$ & $-5.21^{\star}$ & -0.434 & 0.133 & -0.049 & -0.329 & 0.151 & 0.394 & -0.32 & 0.042 & 0.941 \\
\hline$P_{6} \times L_{1}$ & 0.059 & 1.678 & 0.828 & 0.150 & 0.267 & 0.176 & $0.387^{\star}$ & $0.835^{\star}$ & 0.110 & 0.387 & 0.276 & 0.158 \\
\hline $\mathrm{P}_{7} \times \mathrm{L}_{1}$ & $0.270^{\star \star}$ & 4.662 & 1.662 & 0.210 & 0.433 & $0.304^{\star}$ & -0.229 & 0.368 & 0.310 & -0.22 & 0.159 & 0.191 \\
\hline $\mathrm{P}_{8} \times \mathrm{L}_{1}$ & 0.047 & 2.995 & 0.128 & $-0.89 *$ & 0.350 & -0.028 & 0.271 & 0.201 & $-1.47^{*}$ & 0.271 & -0.25 & 0.674 \\
\hline$P_{9} \times L_{1}$ & 0.136 & $11.612^{\star}$ & 4.095 & -0.120 & 0.267 & 0.113 & 0.121 & -0.132 & 0.860 & 0.121 & -0.02 & -0.85 \\
\hline $\mathrm{P}_{10} \times \mathrm{L}_{1}$ & -0.153 & -8.138 & -3.855 & $-1.09 *$ & -0.033 & $-0.29 *$ & -0.163 & -0.249 & $-1.39 *$ & -0.16 & 0.176 & -0.94 \\
\hline$P_{11} \times L_{1}$ & -0.086 & -8.855 & -2.922 & 0.681 & 0.083 & 0.209 & -0.029 & -0.065 & 0.494 & -0.03 & -0.07 & 0.508 \\
\hline$P_{12} \times L_{1}$ & -0.175 & 5.978 & 2.445 & 0.481 & 0.267 & 0.273 & $-0.296^{*}$ & $-0.48^{*}$ & $1.210^{*}$ & -0.29 & -0.29 & -1.15 \\
\hline$P_{13} \times L_{1}$ & 0.175 & -5.738 & -1.538 & $0.992^{\star}$ & 0.000 & 0.255 & 0.121 & -0.215 & -0.240 & 0.121 & 0.142 & -1.12 \\
\hline $\mathrm{P}_{1} \times \mathrm{L}_{2}$ & -0.186 & -5.878 & -1.745 & $0.903^{*}$ & 0.100 & 0.254 & -0.121 & -0.151 & $1.290^{\star}$ & -0.12 & -0.01 & 0.009 \\
\hline $\mathrm{P}_{2} \times \mathrm{L}_{2}$ & $0.275^{\star \star}$ & 4.438 & 0.388 & $-1.28^{\star}$ & $1.100^{\star}$ & $0.310^{\star}$ & $0.279 *$ & $0.649 *$ & -0.610 & 0.279 & 0.274 & -0.84 \\
\hline$P_{3} \times L_{2}$ & 0.069 & -10.495 & -3.578 & 0.276 & -0.067 & 0.049 & -0.054 & 0.082 & -0.710 & -0.05 & -0.01 & -0.31 \\
\hline$P_{4} \times L_{2}$ & 0.130 & 2.905 & 0.572 & 0.083 & $0.633^{*}$ & $0.345^{\star}$ & -0.254 & -0.168 & 0.306 & -0.25 & -0.11 & -0.47 \\
\hline$P_{5} \times L_{2}$ & -0.014 & $13.222^{\star}$ & $5.205^{\star}$ & 0.434 & -0.133 & 0.049 & $0.329 * \star$ & -0.151 & -0.394 & 0.329 & -0.04 & -0.94 \\
\hline$P_{6} \times L_{2}$ & -0.059 & -1.678 & -0.828 & -0.150 & -0.267 & -0.176 & $-0.39 * \star$ & $-0.83^{*}$ & -0.110 & -0.38 & -0.27 & -0.16 \\
\hline $\mathrm{P}_{7} \times \mathrm{L}_{2}$ & $-0.27 \star \star$ & -4.662 & -1.662 & -0.210 & -0.433 & -0.30 * & 0.229 & -0.368 & -0.310 & 0.229 & -0.16 & -0.19 \\
\hline$P_{8} \times L_{2}$ & -0.047 & -2.995 & -0.128 & $0.896^{\star}$ & -0.350 & 0.028 & -0.271 & -0.201 & $1.473^{*}$ & -0.27 & 0.258 & -0.67 \\
\hline$P_{9} \times L_{2}$ & -0.136 & $-11.61^{*}$ & -4.095 & 0.120 & -0.267 & -0.113 & -0.121 & 0.132 & -0.860 & -0.12 & 0.024 & 0.859 \\
\hline $\mathrm{P}_{10} \times \mathrm{L}_{2}$ & 0.153 & 8.138 & 3.855 & $1.091^{\star}$ & 0.033 & $0.294^{\star}$ & 0.163 & 0.249 & $1.390^{*}$ & 0.163 & -0.88 & 0.942 \\
\hline$P_{11} \times L_{2}$ & 0.086 & 8.855 & 2.922 & -0.681 & -0.083 & -0.209 & 0.029 & 0.065 & -0.494 & 0.029 & 0.074 & -0.51 \\
\hline$P_{12} \times L_{2}$ & 0.175 & -5.978 & -2.445 & -0.481 & -0.267 & -0.273 & 0.296 ** & $0.482^{*}$ & $-1.21^{\star}$ & 0.296 & 0.291 & 1.159 \\
\hline $\mathrm{P}_{13} \times \mathrm{L}_{2}$ & -0.175 & 5.738 & 1.538 & $-0.99 *$ & 0.000 & -0.255 & -0.121 & 0.215 & 0.240 & -0.12 & -0.14 & 1.126 \\
\hline
\end{tabular}

\section{Test of epistasis}

The analysis of variance (ANOVA) to test the presence of epistasis for twelve quantitative yield and fiber quality traits is presented in Table 8. The mean squares for the deviations $\left(L_{1 i}+L_{2 i}-G_{i}\right)$ revealed the presence of significant epistasis for all the studied traits except, seed index and fiber reflectance. Farther, partitioning total epistatic effect revealed the presence of highly significant fixable type (additive $\times$ additive) (i) for all studied traits except lint yield, fiber strength and fiber reflectance. Also, the mean square of non-fixable type (additive $\times$ dominance and dominance $\times$ dominance) or $(j+I)$ were highly significant for all the studied traits except seed index, lint index, fiber length, uniformity ratio and 
fiber reflectance. The first type of epistasis additive $x$ additive (i) was found to be much larger in magnitude than the second type additive $x$ dominance $(j+l)$ for all traits. These results indicating that fixable components type of epistasis were more important than non-fixable one for the inheritance of these traits. So, the cotton breeder could use these findings to produce pure line. The same results were reported by ElLawendey et al., 2010 and Saleh 2013.

Jayade et al., 2014 found that standard hybridization and selection procedures could take benefit of fixable epistasis type (i) or (additive $x$ additive). Whereas, non-allelic types of epistasis $(j+l)$ (additive $x$ dominance and dominance $x$ dominance) are not fixable by selection for self pollinated crops. So, the second type of epistasis is not favorable for developing pure lines but could be useful for development hybrid varieties.

The interaction of total two types (i) and $(j+l)$ of epistasis with replications were non-significant for all the studied traits except lint index and fiber length; which indicated that this interaction was not sensitive to the environments (replications). The analysis of variance showed significant mean squares of sums $\left(\mathrm{L}_{1 i}+\mathrm{L}_{2 \mathrm{i}}\right)$ and differences $\left(\mathrm{L}_{1 \mathrm{i}}-\mathrm{L}_{2 \mathrm{i}}\right)$; additive and dominance gene effect, respectively for all the studied traits except lint index for sums while, uniformity ratio and fiber reflectance for differences. These results provide evidence of presence of both additive and dominance gene effect controlling these traits. These results were in line with those of many researchers El-Mansy et al., 2010; 2012 and Mahros, 2016.

The data in Table 8 illustrated that four studied traits out of twelve (boll weight, seed cotton yield, fiber strength and yellowness) had significant mean squares for the both fixable and non-fixable genetic components. Two traits (lint yield and fiber fineness) had significant additive and dominance gene action plus type two of epistasis $(j+l)$. On the other hand, four traits (lint \%, lint index, fiber length and uniformity ratio) showed significant additive and dominance gene action plus type one of epistasis (i). Finally, Table 9 summarizes significant of both fixable and non-fixable gene action over twelve studied traits.

These results are in agreement with those obtained by general and specific combining ability as shown in Tables 5 and 7, respectively. The traits which had significant mean squares for both fixable and non-fixable gene action showed significant GCA and SCA among 15 parents $\left(G_{i}\right)$ and 26 cotton crosses $\left(13 \mathrm{G}_{1 \mathrm{i}} \times \mathrm{L}_{1}\right.$ and $\left.13 \mathrm{G}_{2 \mathrm{i}} \times \mathrm{L}_{2}\right)$. While, two traits seed index and fiber reflectance had non-significant mean squares for both dominance gene effect and specific combining ability over twenty six cotton crosses.

Estimation of both genetic components additive $(D)$, dominance $(H)$, degree of dominance $(H / D)^{0.5}$, direction of dominance $(F)$ and narrow sense heritability $\left(\mathrm{h}^{2}{ }_{\mathrm{ns}}\right)$ are presented in Table 10. Generally, additive genetic component was larger than dominance for all the studied traits. This indicated that additive gene action play an importance role in the inheritance of these traits. So, most of the studied traits had significant general combining ability (GCA) across fifteen parents as presented in Table 5. All these findings could help the Egyptian cotton breeder to use the best selection technique based on the accumulation of additive gene effects to improve Egyptian cotton breeding program to produce pure line.

The degree of dominance $(\mathrm{H} / \mathrm{D})^{0.5}$ were less than unity for all the studied traits, indicating partial or incomplete dominance and certain the fact that, most genes are homozygous and the over-dominance are rare for self pollinated crops. Also, all traits had positive sign, explain that the parent with increasing alleles is dominance than parent with decreasing alleles (El-Mansy et al., 2012 and Dawwam et al., 2016).

Further, the correlation coefficient between the sums $\left(L_{i}+L_{2 i}\right)$ and difference $\left(L_{1 i}-L_{2 i}\right)$ additive and dominance, respectively were found to be insignificant indicating the genes with positive and negative effects were equally distributed among the genotypes including in this study. However, the correlation coefficient for lint index was positive and significant indicating that dominance effect seemed to be acting in one direction. The direction of dominance (F) for twelve yield and fiber quality traits was non-significant which showed that the dominant alleles were dispersed between testers; therefore the two testers did not show any proof of dominance directional for these traits (Table 10).

Generally, the genetic analysis indicated that both additive and non additive types of gene effects were important for most studied traits with the predominant of fixable type of epistasis. Thus for exploitation of all types of gene effects, the intermitting population or recurrent selection followed by progeny test which utilize all kinds of gene effects. 
Table 8

Mean squares for analysis of variance for epistasis $\left(L_{1 i}+L_{2 i}-G_{i}\right)$, sums (additive) $\left(L_{1 i}+L_{2 i}\right)$ and differences (dominance) $\left(L_{1 i}-L_{2 i}\right)$ for all the studied traits

\begin{tabular}{|c|c|c|c|c|c|c|c|}
\hline \multicolumn{8}{|l|}{ Mean squares } \\
\hline \multicolumn{8}{|l|}{ Yield and its components traits } \\
\hline \multirow[t]{2}{*}{ S.o.V } & d.f & Boll weight & Seed cotton yield & Lint yield & Lint \% & Seed index & Lint index \\
\hline & & g & g & g & & g & g \\
\hline i type of epistasis & 1 & $0.460^{\star}$ & $2095.214^{\star}$ & 5.386 & 465.021 ** & 67.954 & $65.788 \star \star$ \\
\hline $\mathrm{j}+\mathrm{I}$ types of epistasis & 12 & 0.470 ** & $1857.922^{\star \star}$ & $320.144 * \star$ & 30.165 & 3.996 & 3.835 \\
\hline Total epistasis & 13 & $0.469 * *$ & $1876.175^{\star \star}$ & $295.931 \star \star$ & 63.616 & 8.916 & $8.600 *$ \\
\hline i type of epistasis $\mathrm{x}$ block & 2 & 0.115 & 523.803 & 1.346 & 116.255 & 16.988 & $16.447^{\star}$ \\
\hline$j+$ I types of epistasis $x$ block & 24 & 0.100 & 472.620 & 58.094 & 41.674 & 5.581 & 2.343 \\
\hline Total epistasis $\mathrm{x}$ block & 26 & 0.101 & 476.557 & 53.729 & 47.419 & 6.458 & 3.428 \\
\hline Additive (D) $\left(\mathrm{L}_{1 \mathrm{i}}+\mathrm{L}_{2 \mathrm{i}}\right)$ & 12 & $0.739 * \star$ & $2896.961 \star \star$ & $453.881^{\star \star}$ & $28.675^{\star \star}$ & 2.210 ** & 3.629 \\
\hline Within families & 24 & 0.041 & 179.141 & 24.643 & 1.160 & 0.263 & 0.091 ** \\
\hline Dominance $(H)\left(L_{1 i}-L_{2 i}\right)$ & 12 & $0.407 * \star$ & $666.526 \star \star$ & $91.287 \star \star$ & $6.790 * \star$ & $2.117^{\star \star}$ & $0.621^{\star *}$ \\
\hline Within families & 24 & 0.039 & 178.619 & 25.364 & 0.698 & 0.249 & 0.118 \\
\hline \multicolumn{8}{|l|}{ Fiber quality traits } \\
\hline s.o.v & d.f & $\begin{array}{l}\text { Fiber } \\
\text { fineness }\end{array}$ & Fiber strength & $\begin{array}{l}\text { Fiber length } \\
\mathrm{mm}\end{array}$ & $\begin{array}{l}\text { Uniformity } \\
\text { ratio }\end{array}$ & Yellowness & $\begin{array}{l}\text { Fiber } \\
\text { reflectance }\end{array}$ \\
\hline & & & & & $\%$ & & \\
\hline i type of epistasis & 1 & 0.954 & $4.017^{\star}$ & $185.850 * \star$ & $210.312^{\star \star}$ & $2.515^{\star}$ & 6.420 \\
\hline $\mathrm{j}+$ I types of epistasis & 12 & $14.169 * \star$ & $30.666^{\star \star}$ & 154.510 & 175.328 & $40.154^{\star \star}$ & 178.444 \\
\hline Total epistasis & 13 & $15.123^{\star *}$ & $34.683^{\star *}$ & $340.360 * \star$ & 385.640 & $42.669 * \star$ & 184.864 \\
\hline i type of epistasis $\mathrm{x}$ block & 2 & 0.2385 & 1.0043 & $46.4625^{\star}$ & 52.578 & 0.6288 & 1.605 \\
\hline$j+$ l types of epistasis $x$ block & 24 & 0.2253 & 0.5516 & 5.2640 & 13.558 & 0.4181 & 12.215 \\
\hline Total epistasis $\mathrm{x}$ block & 26 & 0.2263 & 0.5864 & 8.4332 & 16.559 & 0.4343 & 11.398 \\
\hline Additive (D) $\left(\mathrm{L}_{1 \mathrm{i}}+\mathrm{L}_{2 \mathrm{i}}\right)$ & 12 & $1.161^{\star \star}$ & $1.833^{\star \star}$ & 12.854 ** & $18.252^{\star}$ & $8.449 * \star$ & $48.074 * \star$ \\
\hline Within families & 24 & 0.166 & 0.276 & 1.208 & 6.851 & 0.354 & 12.279 \\
\hline Dominance $(H)\left(L_{1 i}-L_{2 i}\right)$ & 12 & 0.526 ** & $1.208^{\star \star}$ & $10.392 * \star$ & 9.090 & $1.823^{\star \star}$ & 24.036 \\
\hline Within families & 24 & 0.100 & 0.202 & 2.531 & 5.511 & 0.361 & 15.261 \\
\hline
\end{tabular}


Table 9

Summarizes of significant fixable and non-fixable gene action over all the studied traits

\begin{tabular}{|c|c|c|c|c|}
\hline \multirow[t]{2}{*}{ Traits } & \multirow[t]{2}{*}{$\begin{array}{l}\text { Additive (D) } \\
\mathrm{L}_{1 \mathrm{i}}+\mathrm{L}_{2 \mathrm{i}}\end{array}$} & \multirow[t]{2}{*}{$\begin{array}{l}\text { Dominance }(H) \\
L_{1 i}-L_{2 i}\end{array}$} & \multicolumn{2}{|c|}{$\begin{array}{l}\text { Epistasis (i) } \\
\left(L_{1 i}+L_{2 i}-G_{i}\right)\end{array}$} \\
\hline & & & $\mathbf{i}$ & $j+1$ \\
\hline Boll weight & + & + & + & + \\
\hline Seed cotton yield & + & + & + & + \\
\hline Lint yield & + & + & - & + \\
\hline Lint \% & + & + & + & - \\
\hline Seed index & + & + & - & - \\
\hline Lint index & - & + & + & - \\
\hline Fiber fineness & + & + & - & + \\
\hline Fiber length & + & + & + & - \\
\hline Fiber strength & + & + & + & + \\
\hline Uniformity ratio & + & - & + & - \\
\hline Yellowness & + & + & + & + \\
\hline Fiber reflectance & + & - & - & - \\
\hline
\end{tabular}

Table 10

Estimation of genetic components, degree of dominance and narrow sense heritability for the traits studied for all the studied quantitative traits

\begin{tabular}{|c|c|c|c|c|c|c|}
\hline \multicolumn{7}{|c|}{ Yield and its components traits } \\
\hline \multirow[t]{2}{*}{ Genetic components } & Boll weight & Seed cotton yield & Lint yield & Lint $\%$ & Seed index & Lint index \\
\hline & g & g & g & & g & g \\
\hline D & 0.931 & 3623.761 & 572.318 & 36.687 & 2.596 & 4.717 \\
\hline $\mathrm{H}$ & 0.491 & 650.543 & 87.897 & 8.122 & 2.491 & 0.671 \\
\hline$(H / D)^{0.5}$ & 0.726 & 0.424 & 0.392 & 0.471 & 0.980 & 0.377 \\
\hline $\mathbf{F}$ & 0.125 & -1256.028 & -116.742 & 8.097 & 2.084 & 3.302 \\
\hline $\mathbf{r}$ & 0.082 & -0.326 & -0.207 & 0.210 & 0.348 & $0.794^{* *}$ \\
\hline$h^{2} n s$ & 0.6416 & 0.8284 & 0.8491 & 0.8089 & 0.4968 & 0.8653 \\
\hline \multicolumn{7}{|l|}{ Fiber quality traits } \\
\hline \multirow[t]{2}{*}{ Genetic components } & Fiber fineness & Fiber strength & Fiber length & Uniformity & Yellowness & Fiber reflectance \\
\hline & & & $\mathrm{mm}$ & Ratio \% & & \\
\hline D & 1.327 & 2.076 & 15.527 & 15.201 & 10.793 & 47.727 \\
\hline $\mathrm{H}$ & 0.569 & 1.342 & 10.481 & 4.772 & 1.949 & 11.700 \\
\hline$(H / D)^{0.5}$ & 0.655 & 0.804 & 0.822 & 0.560 & 0.425 & 0.495 \\
\hline F & -0.515 & 1.690 & -3.617 & -6.469 & -0.162 & -1.825 \\
\hline $\mathbf{r}$ & -0.238 & 0.410 & -0.113 & -0.181 & -0.015 & -0.019 \\
\hline$h_{n s}^{2}$ & 0.6791 & 0.5868 & 0.5738 & 0.7589 & 0.8349 & 0.7452 \\
\hline
\end{tabular}


The type and direction of association, which may occur between the studied traits, is important for selection techniques. The plant breeder

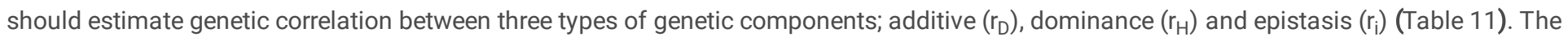
obtained results provide that increasing additive gene effects controlling boll weight was controlled the increasing ones controlling yield and its components traits. Makhdoom et al., 2010 reported that boll weight is the independent key for yield components and played a prime role in managing seed cotton yield. These results are in agreement with Farooq et al., 2014 and El-Mansy 2015. Lint yield and lint \% were additively correlated with seed index and lint index. On the other hand, fiber length has the same direction with two fiber traits; fiber strength and uniformity ratio.

The dominance genetic correlation was positive and significant between seed index and both boll weight and lint index. While, significant and negative dominance correlation was observed between lint \% and fiber strength, yellowness and fiber reflectance. Regarding the results of epistasis genetic correlation recorded positive and significant correlation only between yield and its components (seed cotton yield, lint index and seed index). Also, genetic significant and positive epistasis controls both fiber traits; fiber length and uniformity ratio.

All the findings obtained from both levels of genetic correlation prove that GCA and SCA controlled by both additive and dominance gene effect, respectively. If epistasis is present the different types of non-allelic interaction (i) or $(j+I)$ will control both GCA and SCA, respectively. So, the results obtained from Tables 5, 7, 9 and 10 are in the same trend. These results may help cotton breeder how to improve cotton yield, which is a quantitative traits with low or moderate heritability values and additive gene effect is a fixable type. The improvement can be effective by using indirect selection for its components like; lint yield, lint \% and lint index and lint \% which had high narrow sense heritability $84.91 \%$, $80.89 \%$ and $86.53 \%$, respectively (Table 10). The advantages of triple test cross matting design are break up undesirable linkage groups to obtain new recombinant lines, provide information about fixable and non-fixable genetic components and the genetic correlation between genetic components.

Finally, from the previous results it may be concluded that the studied traits were controlled by additive and additive $x$ additive (i) type of epitasis and most desirable correlation were due to additive gene effects and few cases of non additive type. This suggests a common genetic pool, pleiotropy or linkage. The appearance of epistasis components in early generations is not favorable to the plant breeder because selection produces will be not fruitful, so selection process should be delay to late generations. Thus recurrent selection or intermitting population may be useful in the presence of epistasis because these techniques will increase frequency of favorable alleles. 
Table 11

Genotypic correlation between additive $\left(r_{D}\right)\left(L_{1 i}+L_{2 i}\right)$, dominance $\left(r_{H}\right)\left(L_{1 i}-L_{2 i}\right)$ and epistasis $\left(r_{i}\right)\left(L_{1 i}+L_{2 i}-G_{i}\right)$, gene effects controlling both yield, its components and fiber quality traits

\begin{tabular}{|c|c|c|c|c|c|c|c|c|c|c|c|c|c|}
\hline \multirow[t]{2}{*}{ Traits } & \multirow[t]{2}{*}{$r$} & \multirow{2}{*}{$\begin{array}{l}\text { BW } \\
\text { g }\end{array}$} & \multirow{2}{*}{$\begin{array}{l}\text { SCY } \\
\text { g }\end{array}$} & \multirow{2}{*}{$\begin{array}{l}\text { LY } \\
\text { g }\end{array}$} & \multirow[t]{2}{*}{ L\% } & \multirow{2}{*}{$\begin{array}{l}\text { SI } \\
\text { g }\end{array}$} & \multirow{2}{*}{$\begin{array}{l}\text { LI } \\
\text { g }\end{array}$} & \multirow[t]{2}{*}{ FF } & \multirow[t]{2}{*}{ FS } & \multirow{2}{*}{$\begin{array}{l}\mathrm{FL} \\
\mathrm{mm}\end{array}$} & \multirow[t]{2}{*}{ UR\% } & \multirow[t]{2}{*}{$+b$} & \multirow[t]{2}{*}{ RD } \\
\hline & & & & & & & & & & & & & \\
\hline \multirow{3}{*}{$\begin{array}{l}\text { BW } \\
\text { g }\end{array}$} & $r_{D}$ & 1.000 & $0.541^{*}$ & $0.616^{\star}$ & $0.596^{*}$ & $0.611^{*}$ & 0.660 ** & $0.562^{\star}$ & -0.284 & 0.024 & 0.162 & -0.149 & -0.291 \\
\hline & $r_{H}$ & 1.000 & 0.187 & 0.092 & -0.315 & $0.588^{*}$ & 0.377 & 0.256 & $0.651^{*}$ & -0.013 & 0.303 & 0.527 & -0.271 \\
\hline & $r_{i}$ & 1.000 & -0.422 & -0.361 & 0.342 & -0.013 & -0.351 & -0.055 & 0.155 & 0.062 & -0.249 & 0.211 & 0.317 \\
\hline \multirow{3}{*}{$\begin{array}{l}\text { SCY } \\
\text { g }\end{array}$} & $I_{D}$ & & 1.000 & $0.984^{\star \star}$ & 0.200 & $0.488^{*}$ & 0.341 & 0.184 & -0.127 & $0.482^{\star}$ & 0.424 & -0.273 & -0.140 \\
\hline & $r_{H}$ & & 1.000 & 0.974 ** & -0.022 & 0.346 & 0.348 & 0.341 & -0.007 & 0.186 & 0.450 & -0.109 & -0.173 \\
\hline & $r_{i}$ & & 1.000 & 0.850 ** & -0.004 & -0.036 & 0.362 & -0.165 & 0.009 & -0.043 & -0.122 & 0.335 & -0.298 \\
\hline \multirow{3}{*}{$\begin{array}{l}\text { LY } \\
\text { g }\end{array}$} & $r_{D}$ & & & 1.000 & 0.367 & $0.555^{\star}$ & $0.486^{\star}$ & 0.294 & -0.132 & 0.435 & 0.407 & -0.293 & -0.137 \\
\hline & $r_{H}$ & & & 1.000 & 0.190 & 0.189 & 0.360 & 0.293 & -0.134 & 0.272 & 0.471 & -0.167 & -0.151 \\
\hline & $r_{i}$ & & & 1.000 & -0.037 & 0.226 & 0.544 & -0.151 & 0.020 & 0.329 & 0.157 & 0.280 & -0.310 \\
\hline \multirow[t]{3}{*}{ L\% } & $\mathrm{r}_{\mathrm{D}}$ & & & & 1.000 & $0.579 *$ & $0.937^{\star \star}$ & $0.693^{\star \star}$ & -0.121 & -0.231 & -0.052 & -0.221 & -0.001 \\
\hline & $r_{H}$ & & & & 1.000 & -0.428 & 0.367 & -0.277 & $-0.576^{\star}$ & 0.451 & 0.223 & -0.331 & 0.070 \\
\hline & $r_{i}$ & & & & 1.000 & 0.187 & -0.076 & -0.184 & -0.425 & -0.138 & -0.024 & $0.665^{\star}$ & -0.327 \\
\hline \multirow{3}{*}{$\begin{array}{l}\text { SI } \\
\text { g }\end{array}$} & $r_{D}$ & & & & & 1.000 & $0.826^{\star *}$ & $0.697^{\star *}$ & 0.016 & -0.070 & -0.044 & 0.048 & -0.035 \\
\hline & $r_{H}$ & & & & & 1.000 & $0.682^{\star}$ & 0.032 & 0.432 & 0.020 & 0.332 & 0.164 & -0.252 \\
\hline & $r_{i}$ & & & & & 1.000 & $0.831^{\star \star}$ & 0.080 & 0.025 & $0.627^{\star}$ & 0.372 & 0.013 & 0.253 \\
\hline \multirow{3}{*}{$\begin{array}{l}\text { LI } \\
\text { g }\end{array}$} & $\mathrm{r}_{\mathrm{D}}$ & & & & & & 1.000 & $0.774^{\star \star}$ & -0.073 & -0.186 & -0.065 & -0.111 & 0.002 \\
\hline & $r_{H}$ & & & & & & 1.000 & -0.166 & -0.008 & 0.366 & 0.517 & -0.066 & -0.236 \\
\hline & $r_{i}$ & & & & & & 1.000 & 0.062 & 0.102 & $0.701^{\text {** }}$ & 0.431 & -0.076 & 0.084 \\
\hline \multirow[t]{3}{*}{ FF } & $r_{D}$ & & & & & & & 1.000 & 0.276 & -0.011 & -0.027 & -0.203 & 0.258 \\
\hline & $r_{H}$ & & & & & & & 1.000 & 0.341 & -0.451 & -0.153 & 0.029 & 0.008 \\
\hline & $r_{i}$ & & & & & & & 1.000 & 0.431 & 0.266 & 0.254 & -0.483 & 0.047 \\
\hline \multirow[t]{3}{*}{ FS } & $r_{D}$ & & & & & & & & 1.000 & $0.507^{\star}$ & 0.394 & 0.008 & 0.093 \\
\hline & $r_{H}$ & & & & & & & & 1.000 & -0.197 & -0.023 & 0.188 & 0.324 \\
\hline & $r_{i}$ & & & & & & & & 1.000 & 0.154 & 0.059 & -0.176 & 0.201 \\
\hline \multirow{3}{*}{$\begin{array}{l}\mathrm{FL} \\
\mathrm{mm}\end{array}$} & $I_{D}$ & & & & & & & & & 1.000 & $0.82^{\star \star}$ & -0.093 & -0.177 \\
\hline & $r_{H}$ & & & & & & & & & 1.000 & $0.81^{\star \star}$ & 0.038 & -0.043 \\
\hline & $r_{i}$ & & & & & & & & & 1.000 & $0.606^{*}$ & -0.240 & -0.015 \\
\hline \multirow[t]{3}{*}{ UR\% } & $r_{D}$ & & & & & & & & & & 1.000 & -0.256 & $-0.489 *$ \\
\hline & $r_{H}$ & & & & & & & & & & 1.000 & 0.137 & -0.202 \\
\hline & $r_{i}$ & & & & & & & & & & 1.000 & -0.033 & -0.284 \\
\hline
\end{tabular}

* and ** significant at $5 \%$ and $1 \%$ levels of probability, respectively. 


\begin{tabular}{|c|c|c|c|c|c|c|c|c|c|c|c|c|c|}
\hline Traits & $r$ & $\begin{array}{l}\text { BW } \\
\text { g }\end{array}$ & $\begin{array}{l}\text { SCY } \\
\mathrm{g}\end{array}$ & $\begin{array}{l}\text { LY } \\
\text { g }\end{array}$ & L\% & $\begin{array}{l}\text { SI } \\
\text { g }\end{array}$ & $\begin{array}{l}\text { LI } \\
\mathbf{g}\end{array}$ & FF & FS & $\begin{array}{l}\mathrm{FL} \\
\mathrm{mm}\end{array}$ & UR\% & $+b$ & RD \\
\hline \multirow[t]{3}{*}{$+b$} & $\mathrm{r}_{\mathrm{D}}$ & & & & & & & & & & & 1.000 & -0.110 \\
\hline & $r_{H}$ & & & & & & & & & & & 1.000 & $-0.74^{\star \star}$ \\
\hline & $r_{i}$ & & & & & & & & & & & 1.000 & -0.306 \\
\hline
\end{tabular}

\section{Genetic diversity}

Genetic divergence studies in cotton revealed some interesting features of differentiation and adaptability such as cluster analysis which provide additional information for studding interrelationship between genotypes and giving graphical assessment of genetic variability. For this propose hierarchical cluster analysis, on the basis of Wards method and interval Euclidean distance, was applied to investigate genetic distance and diversity between the fifteen parental cotton genotypes. The data matrix of the dissimilarity coefficient on the basis of Euclidean distance is presented in Table 12. This dissimilarity coefficient was ranged from 2.93 between Giza 70 and Giza 76 to 43.45 between Giza 80 and Giza 88. This wide range of genetic distance among these genotypes reflected the presence of wide range of genetic variation and provides an opportunity to improve the cotton genetic basis by implementing crossing technique.

Cluster analysis sequestrated the fifteen cotton genotypes into nine major groups on the basis of dissimilarity and contributed the studied traits as shown in Fig. 1. It's clear that the two testers were grouped into different clusters with average dissimilarity coefficient 14.2. These female parents varied in general combing ability for most studied traits. On the other side, the commercial variety Giza 86 formed a unique cluster and wide distance from the other parents (Table 13). So, the commercial variety Giza 86 was classified as the best combiner for all yield traits (Table 5). The half sense parents; Giza 89 and Giza 89 × S6 were grouped at the same cluster with narrow genetic distance. On the other side, Giza 77 grouped in a cluster and characterized as a good combiner for most yield traits.

The five genotypes (Giza 70, Giza 76, Ashmouni, Giza 81 and Alexandria 4) were grouped together with narrow genetic base. These genotypes described as poor general combiners for most studied yield traits but showed some sort of superior fiber quality traits. In this trend, El-Mansy, 2014, Abd El-Moghny et al., 2015 a and b and Akter et al., 2019 used phenotypic performance to classified cotton genotypes into different clusters.

Genotypes grouped in the same cluster (intra-cluster) are expected to be genetically similar than genotypes grouped in different clusters (intercluster) (Table 13). These results indicated wide genetic distance among fifteen parental cotton genotypes. The highest inter-cluster distance was observed between clusters 6,8 followed by clusters 2,8 and 3, 2, respectively. While, the lowest genetic distance occurred between clusters 9,5 followed by clusters 1,4 and clusters 2,3 . So, hybridization between clusters is more useful than within clusters to increase genetic variability and obtain more transgreesive segregant in early generations. The same findings were obtained by Abd El-Moghny et al., 2015 (a). Machado et al., 2002 noticed that choosing parents is very important step to obtain the best combination. The parents not only had higher genetic divergence but also have higher mean performance of parents and their $F_{1}$ s. In addition to GCA and SCA effects are more informative than mean performance values (Abd El-Salam et al. 2010 and El-Mansy et al., 2014). 
Genetic diversity between thirteen cotton genotypes $\left(\mathrm{G}_{\mathrm{i}}\right)$ and the two testers $\left(\mathrm{L}_{1}\right.$ and $\left.\mathrm{L}_{2}\right)$ cotton genotypes

\begin{tabular}{|c|c|c|c|c|c|c|c|c|c|c|c|c|c|c|c|}
\hline \multirow[t]{2}{*}{$G_{i}$} & \multicolumn{15}{|c|}{ Euclidean Distance } \\
\hline & $\mathrm{P}_{1}$ & $\mathrm{P}_{2}$ & $P_{3}$ & $\mathrm{P}_{4}$ & $P_{5}$ & $\mathrm{P}_{6}$ & $\mathrm{P}_{7}$ & $\mathrm{P}_{8}$ & $P_{9}$ & $\mathrm{P}_{10}$ & $P_{11}$ & $\mathrm{P}_{12}$ & $\mathrm{P}_{13}$ & $\mathrm{~L}_{1}$ & $\mathrm{~L}_{2}$ \\
\hline$P_{1 i}$ & 0.00 & 16.87 & 15.16 & 9.06 & 13.62 & 12.10 & 9.23 & 29.51 & 17.84 & 19.93 & 6.53 & 9.97 & 17.00 & 16.44 & 6.42 \\
\hline $\mathrm{P}_{2}$ & & 0.00 & $27.13^{\star}$ & 20.30 & 28.36 & 26.93 & 22.76 & 42.90 & 31.31 & 9.53 & 16.54 & 21.55 & 7.44 & 29.67 & 17.16 \\
\hline$P_{3}$ & & & 0.00 & 10.11 & 6.94 & 7.14 & 11.22 & 18.65 & 5.70 & 30.61 & 11.95 & 6.45 & 24.14 & 7.11 & 14.45 \\
\hline $\mathrm{P}_{4}$ & & & & 0.00 & 9.86 & 8.76 & 9.48 & 26.00 & 14.13 & 24.02 & 8.54 & 6.38 & 19.35 & 12.63 & 9.25 \\
\hline$P_{5}$ & & & & & 0.00 & 2.93 & 9.14 & 18.09 & 7.17 & 31.12 & 12.70 & 8.10 & 26.54 & 6.33 & 13.30 \\
\hline$P_{6}$ & & & & & & 0.00 & 9.57 & 20.49 & 8.58 & 30.39 & 11.68 & 7.80 & 25.45 & 8.54 & 12.58 \\
\hline$P_{7}$ & & & & & & & 0.00 & 21.60 & 12.26 & 23.61 & 8.58 & 7.15 & 21.06 & 8.95 & 6.83 \\
\hline$P_{8}$ & & & & & & & & 0.00 & 13.74 & 43.57 & 27.39 & 22.30 & 39.76 & 13.94 & 27.23 \\
\hline$P_{9}$ & & & & & & & & & 0.00 & 33.90 & 15.39 & 10.12 & 28.37 & 5.34 & 16.94 \\
\hline$P_{10}$ & & & & & & & & & & 0.00 & 19.72 & 24.50 & 11.33 & 31.31 & 19.07 \\
\hline$P_{11}$ & & & & & & & & & & & 0.00 & 6.15 & 14.28 & 14.09 & 6.62 \\
\hline$P_{12}$ & & & & & & & & & & & & 0.00 & 18.97 & 8.96 & 8.95 \\
\hline$P_{13}$ & & & & & & & & & & & & & 0.00 & 26.98 & 16.97 \\
\hline $\mathrm{L}_{1}$ & & & & & & & & & & & & & & 0.00 & 14.20 \\
\hline $\mathrm{L}_{2}$ & & & & & & & & & & & & & & & 0.00 \\
\hline
\end{tabular}

Table 13

Average divergence values for intra (Diagonal values) and inter (Above diagonal) clusters between nine clusters for the fifteen cotton genotypes for twelve1 quantitative characteristic

\begin{tabular}{|llllllllll|}
\hline Clusters & $\mathbf{1}$ & $\mathbf{2}$ & $\mathbf{3}$ & $\mathbf{4}$ & $\mathbf{5}$ & $\mathbf{6}$ & $\mathbf{7}$ & $\mathbf{8}$ & $\mathbf{9}$ \\
\hline $\mathbf{1}$ & 3.766 & 16.433 & 15.687 & 7.210 & 12.020 & 19.214 & 7.364 & 27.810 & 14.354 \\
$\mathbf{2}$ & & 0.000 & 7.442 & 20.693 & 27.616 & 9.530 & 22.759 & 42.897 & 29.207 \\
$\mathbf{3}$ & & & 0.000 & 18.892 & 25.963 & 11.334 & 21.065 & 39.758 & 26.320 \\
$\mathbf{4}$ & & & & 3.191 & 7.927 & 24.049 & 7.769 & 24.011 & 9.575 \\
\hline $\mathbf{5}$ & & & & & 1.464 & 30.722 & 9.240 & 19.272 & 6.454 \\
$\mathbf{6}$ & & & & & & 0.000 & 23.606 & 43.567 & 31.778 \\
$\mathbf{7}$ & & & & & & & 0.000 & 21.595 & 10.313 \\
$\mathbf{8}$ & & & & & & & & 0.000 & 15.208 \\
\hline $\mathbf{9}$ & & & & & & & & & 3.489 \\
\hline
\end{tabular}

\section{Conclusions}

This investigation was designed to use modified triple test cross analysis to investigate type of gene actions and genetic correlation between most quantitative economic traits in cotton for rightful decision about effective selection procedures. Also, cotton breeder need to increase genetic diversity among new varieties and maintaining the complexity between desired traits which will present in commercial varieties. So, developing different traits combinations is a difficult process as the introgression of new genetic materials is a result of disturbing genes responsible for desired traits. The use of crosses between divergent parents could be meaning to achieve combining ability. 


\section{Abbreviations}

BW: boll weight

SCY/P: seed cotton yield / plant

LY/P: lint yield / Plant

L\%: lint percentage

FL: Fiber length

FF: fiber fineness (micronaire value)

FS: fiber strength (Presley index)

UR\%: uniformity ratio

+b: yellowness

RD: Fiber reflectance

TTC: triple test cross analysis

GCA: general combining ability

SCA: specific combining ability

D: additive genetic variance

$\mathrm{H}$ : dominance genetic variance

i: epistasis genetic variance

\section{Declarations}

Acknowledgments:-

The authors are grateful to Cotton Research Institute (CRI), Agricultural Research Center (ARC), Egypt for funding this research.

Authors' contributions:-

Yasser M. El-Mansy and Ahmed M. Abdelmoghny, provided cotton germplasm and parents evaluation for the first year, data analyzed, interpreted the data, wrote the manuscript and edit for publication. Reham H. A. Gibely and Adel H. Mabrouk making 26 cotton crosses, designed parents and crosses experiment, monitored field experiment, laboratory work and help to revise the manuscript. All authors read and approved the final manuscript and have made substantive intellectual contributions to the manuscript.

Funding Funds for this research:-

Fund for this research was provided by Cotton Research Institute (CRI), Agricultural Research Center (ARC), Egypt.

Availability of data and materials:-

All data generated or analyzed during this study are included in this published article.

\section{Ethics approval and consent to participate:-}

Not applicable.

Consent for publication:-

All authors have consent for submission of manuscript.

Competing interests:-

Page $22 / 25$ 


\section{References}

1. Abd El-Moghny, A. M.; S. Max, Mariz and H. A. Gibely, Reham. 2015 a. Nature of genetic divergence among some cotton genotypes. J. of Cotton Science 19:368-374.

2. Abd El-Moghny, A. M.; S. Max, Mariz and H. A. Gibely, Reham. 2015 b. Genetic variation and associations between some agronomic, chemical seed components and seedling vigor characters in some cotton varieties. Bull. Fac .Agric., Cairo Univ. 66: $390-399$.

3. AL-Hibbiny, Y. I. M. 2015. Estimation of heterosis, combining ability and gene action by using Line x Tester analysis in cotton(Gossypium barbadense) Egypt. J. Plant Breed. 19(2):385 -405.

4. AL-Hibbiny, Y. I. M.; A. H. Mabrouk and Badeaa A. Mahmoud. 2019. Line x tester analysis for yield components and fiber properties in some cotton crosses of(Gossypium barbadense). Menoufia J. Plant Prod. 4:505-5025.

5. Abd El-Salam, M. E.; Y. M. El-Mansy and Rokia M. Hassan. 2010. The relative importance of characters affecting genetic divergence in cotton. J. Agric. Kafer El-Sheikh Univ. 36(1): 44-63.

6. Akter, T.; A. K. M. A. Islam; M. G. Rasul; S. Kundu; Khalequzzaman and J. U. Ahmed. 2019. Evaluation of genetic diversity in short duration cotton(Gossypium hirsutum). J. of Cotton Research, 2(1): 2-6.

7. Başbağ, S. ; R. Ekinci and O. Gencer. 2007. Combining ability and heterosis for earliness characters in L x T population of hirsutumL. Hered., $144: 185-190$.

8. Dawwam, H. A.; F. A. Hendawy; M. A. Abd El-Aziz; R. M. Esmail A. B. Khatab and Mahros, H. El-Shymaa. 2016.Using triple test cross techinque for partitioning the components of genetic variance and predicing the properties of new recombinant inbred lines in cotton ( barbadense L.). 10 ${ }^{\text {th }}$ International Plant Breeding Conference, 5-6 Septmber, Fac. Agric. Menoufia Univ.

9. Determination of genetic components

10. through triple test cross in cotton (G. barbadense

11. ). J. Agric. Res. Kafer El-Sheikh Univ. 36:

12. $240-257$.

13. El-Lawendey, M.M., Y.M. El-Mansy and M.E. Abd El-Salam.

14. Determination of genetic components

15. through triple test cross in cotton (G. barbadense

16. ). J. Agric. Res. Kafer El-Sheikh Univ. 36:

17. 240-257.

18. El-Lawendey, M.M., Y.M. El-Mansy and M.E. Abd El-Salam.

19. Determination of genetic components

20. through triple test cross in cotton (G. barbadense

21.). J. Agric. Res. Kafer El-Sheikh Univ. 36:

22. 240-257.

23. El-Lawendey, M.M., Y.M. El-Mansy and M.E. Abd El-Salam.

24. Determination of genetic components

25. through triple test cross in cotton (G. barbadense

26. ). J. Agric. Res. Kafer El-Sheikh Univ. 36:

27. 240-257.

28. Ekinci, R. and S. Başbağ. 2015. Combining ability for yield and its components in diallel crosses of cotton. Not. Sci. Biol., 7(1):72-80.

29. El-Lawendey, M. M., Y. M. El-Mansy and M. E. Abd El-Salam. 2010. Determination of genetic components through triple test cross in cotton ( barbadenseL.). J. Agric. Res. Kafer El-Sheikh Univ. 36: 240-257.

30. El-Mansy, Y. M.; W. M. B. Yehia and M. A. El-Dahan. 2010.Role of epistasis in the inheritance of characters related to earliness in cotton. Minufia, J. Agric. Res. 35(2):635-648.

31. El-Mansy, Y. M.; M. M. El-Lawendey; and M. A. A. El-Dahan. 2012. Genetic variability in growth habit and development of boll and its relation with yield and fiber characters in Egyptian cotton. Egypt. J. Plant Breed., 16(1): 83-97. 
32. El-Mansy, Y. M.; M. M. E. Abdel-Salam and B. M. Ramdan. 2014. Multivariate analysis of genetic divergence and combining ability in Gossypium barbadensel. Agric. Res. Kafer El-Sheikh Univ. 40 (1): 85-103.

33. El-Mansy, Y. M. 2015. Impact of mating system on genetic variability and correlation coefficients in cotton( barbadense L.)). Minufiya J. Agric. Res. 40(1): 119-129.

34. Farooq, J.; M. Anwar, M. Riaz; A. Farooq and F. Ilahi. 2014. Correlation and path coefficiency analysis of earliness, fiber uality and yield contributing traits in cotton hirsutum L. J. Anim. And Plant Sci., 24(3): 781-790.

35. Fasahat, P.; A. Rajabi; J. M. Rad and J. Derera. 2016.Principles and utilization of combining ability in plant breeding. Biom. Biostat. Int. J. $4(1): 1-22$.

36. Gomez, K. A. and A. A. Gomez. 1984. Statistical Procedures for Agricultural Research. $2^{\text {nd }}$ Ed John Wiley and sons, NewYork, USA.

37. Jayade, V. S.; S.R. Patil; P.D. Peshattiwarand and R. D. Deotale. 2014. Simplified triple test cross analysis for yield, yieldcontirbting and fiber traits in cotton (Gossypium hirsutum L.).). International Journal of Researches In Biosciences, Agriculture \& Technology, 2 (II) : $177-187$.

38. Johnson, R. A. and D. W. Wichern. 1998. Applied Multivariate Statistical Analysis. $4^{\text {th }}$ Prentice-Hall, Upper Saddle River, New Jersey, USA.

39. Jinks, J. L. and D. S. Virk. 1977. A modified triples test-cross analysis to test and allow for inadequate testers. Heredity, 39 (1): $165-170$.

40. Jinks, J. L.; J. M. Perkins and E. L. Breese. 1969.A general method of detecting additive, dominance and epistatic variation for metric traits. II. Application to inbreed lines. Heredity, 24: 45-47.

41. Kempthorne, 0. 1957. An Introduction to Genetic Statstics. JohnWiley and Sons Inc. New York.

42. Ketata, H.; E. L. Smith; L. K. Edwards and R. W. McNew. 1976. Detection of epistasis, additive and dominance variation in winter wheat. Crop Sci., 16: 1-4.

43. Khattak, G. S. S.; M. A. Haq; M. Ashraf; A. J. Khan and R. Zamir. 2002. Genetic architecture of secondary yield components in mungbean(Vigna radiata) Wilczek. Breeding, Sci., 52: 235-241.

44. Machado, C. F.; G. H. S. Munes and D. F. Ferreria. 2002. Genetic divergence among genotypes using multivariate technique. Ciencia Rural, 32(2):251-258.

45. Mahros, H. El-Shymaa. 2016. Inhertance of seed cotton yield and its components using triple test cross analysis in some cotton crosses. Ph.D. Fac. Agric. Menoufia Univ.

46. Mahrous, H. 2018. Line $\times$ tester analysis for yield and fiber quality traits in Egyptian cotton under heat conditions. J. Plant Production, Mansoura Univ., Vol. 9 (6): 573 - 578.

47. Makhdoom, K.; N. U. Khan; S. Batool; Z. Bibi; Farhatullah; S. Khan; F. Mohammad; D. Hussain; Raziuddin; M. Sajjad and N. Khan. 2010. Genetic aptitude and correlation studies in Gossypium hirsutumL. Pak. J. Bot., 42(3): 2011-2017.

48. Saleh, M. R. M. Eman. 2013. Genetic estimation of yield and yield components in cotton through triple test cross analysis. J. Plant Production, Mansoura Univ., 4 (2): 229 - 237.

49. Sharma, J. R. 1988. Statistical and Biometrical Techniques in Plant Breeding. New Delhi, New Age International.

50. Singh, R. K. and B. D. Chaudhary. 1985. Biometrical Methods in Quantitative Genetics and Analysis. Kalyani Publ., New Delhi.

51. Sing, P. and S. S. Narayanan, 2013. Biometrical Techniques in Quantitative Genetics in Plant Breeding. Kalyani Publ., $5^{\text {th }}$ New Delhi.

52. Sultan, M. S.; M. A. Abdel-Moneam; Y. M. EL-Mansy and Huda S. El-Morshidy. 2018.Estimating of heterosis and combining ability for some Egyptian cotton genotypes using line x tester mating design. J. Plant Production, Mansoura Univ., Vol. 9 (12): $1121-1127$.

53. Soliman, Y. A. M.; Y. M. El-Mansy and M. A. M. Allam. 2008. Triple test cross analysis of some economic characters in cotton G. barbadense. Egypt .J. of Appl. Sci., 23 (4A): 125-137.

54. Yehia, W. M. B. and E. F. El-Hashash. 2019.Combining ability effects and heterosis estimates through line $x$ tester analysis for yield, yield components and fiber traits in Egyptian cotton. J. Agro. Technology and Engineering Management, 2(2): $248-262$.

\section{Figures}




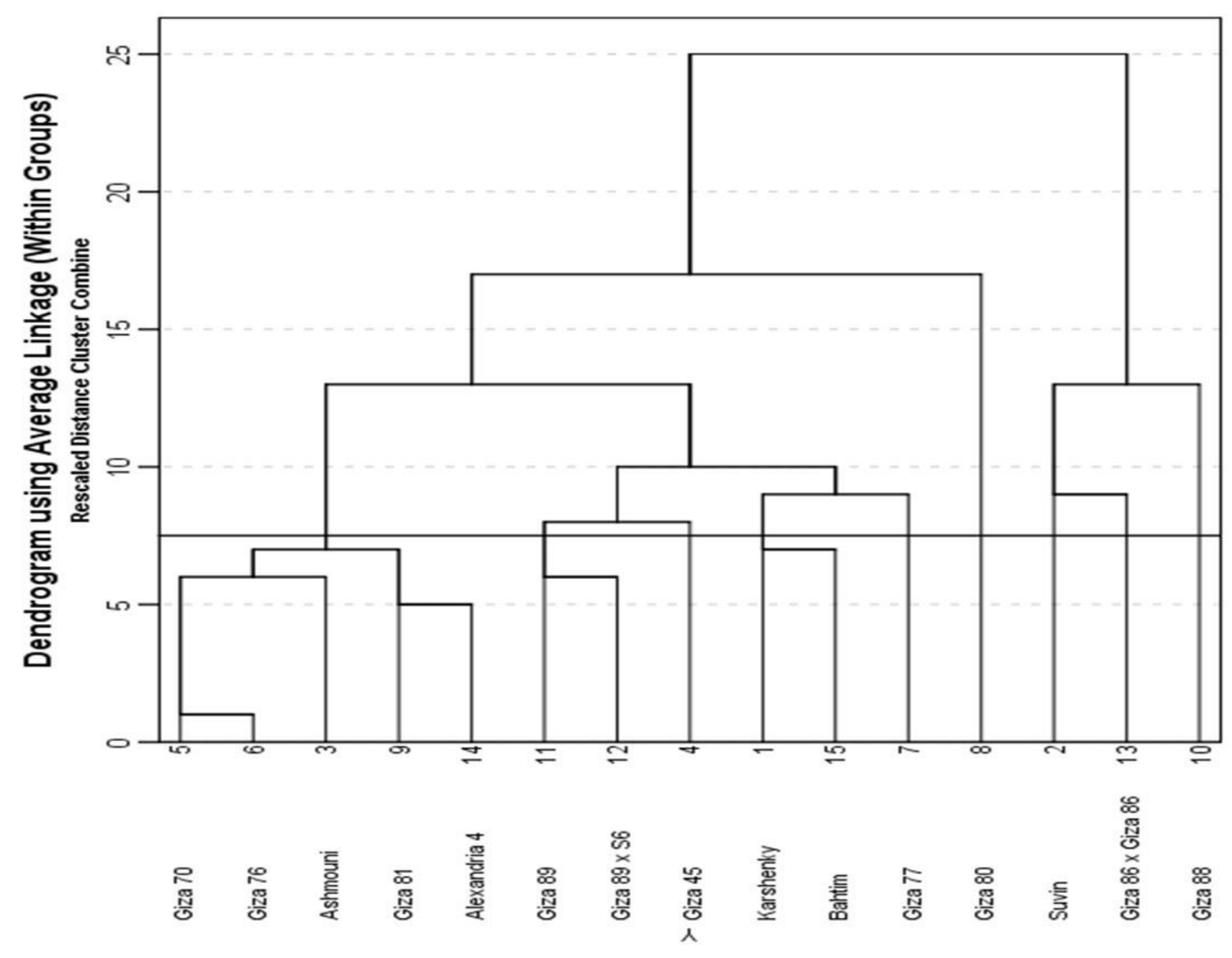

Figure 1

Dendrogram for the fifteen cotton genotypes using average linkage (within groups) 